\title{
Research Paper: \\ Inequality in the Utilization of Rehabilitation Services Among Urban and Rural Households in Iran: A Cross-Sectional Study
}

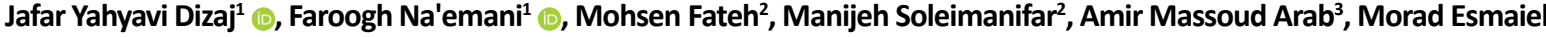 \\ Zali $^{2}$, Shahin Soltani ${ }^{4}{ }^{\circ}$, *Ali Kazemi Karyani ${ }^{4}$ (1)
}

1. Department of Health Management and Economics, School of Public Health, Tehran University of Medical Sciences, Tehran, Iran. 2. Lifestyle and Health Management Research Group, Academic Center for Education, Culture and Research (ACECR), Tehran, Iran.

3. Department of Physiotherapy, School of Rehabilitation Sciences, University of Social Welfare and Rehabilitation Sciences, Tehran, Iran.

4. Research Center for Environmental Determinants of Health, Health Institute, Kermanshah University of Medical Sciences, Kermanshah, Iran.

\begin{tabular}{l|l}
$\begin{array}{c}\text { Use yur device to scan } \\
\text { and read the article online }\end{array}$ \\
Utilization of Rehabilitation Services Among Urban and Rural Households in Iran: A Cross-Sectional Study (Persian)]. Archives \\
of Rehabilitation. 2020; 21(3):336-357. https://doi.org/10.32598/RJ.21.3.2893.1 \\
dol'https://doi.org/10.32598/RJ.21.3.2893.1
\end{tabular}

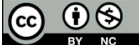

Received: 10 Dec 2019 Accepted: 05 Feb 2020 Available Online: 01 Oct 2020

Keywords:

Utilization, Rehabilitation services, Income inequality, Concentration index, Iran

\begin{abstract}
Objective Demographic and epidemiological changes in the $21^{\text {st }}$ century have created new challenges such as aging and the rising trend of non-communicable diseases. The high prevalence of disability (due to the growing aging population), non-communicable diseases, and accidents have increased the demand for rehabilitation services. However, there are not enough resources to meet all current needs in many parts of the world. The purpose of this study is to investigate the inequality in the utilization of rehabilitation services between Iranian households in urban and rural areas. Materials \& Methods This is a descriptive-analytical study with a cross-sectional design using the household expenditure, income survey data from the Statistics Center of Iran in 2018. The used instrument was a questionnaire surveying the expenditure and income of urban and rural households (social characteristics of household members, place of residence and main living facilities, food/non-food expenses, and household income), which was completed through interviewing the household head or a member over 15 years of age. The study samples were 18610 households in rural areas and 20348 households in urban areas. After extracting and refining the data, 38958 households were included in the study. Factors affecting their utilization of rehabilitation services and the inequality in utilization were analyzed using the Chi-square test and the Concentration Index $(\mathrm{Cl})$, respectively. Data were extracted in MS Access 2013 and MS Excel 2013 applications and were analyzed in STATA V.14.1 software. The geographic distribution of the service utilization was plotted using ArcGIS Map V. 10 software.

Results A total of 258 households (0.77\%) used rehabilitation services. Of these, $226(87 \%)$ had a male head, and 32 (13\%) had a female head. About 60\% had 3-4 members, and 239 (92\%) had insurance coverage, and others (8\%) had no insurance coverage. Also, $173(67 \%)$ had an employed head. Finally, 55\% were living in rural areas, and $45 \%$ in urban areas. Uninsured households had less use of rehabilitation services $(\mathrm{P}<0.05)$. Also, $1.32 \%$ of the fifth income quintile (highest income) used rehabilitation services, while this rate was $0.35 \%$ for households in the first quintile $(P<0.001)$. Zanjan, Qazvin, Khuzestan, Isfahan, Lorestan, Bushehr, and Semnan provinces had the lowest service utilization rates in urban/rural areas and the whole country. Qom Province had a better status regarding service utilization in urban areas, while East Azerbaijan, Mazandaran, Golestan, Yazd, Fars, and Hormozgan provinces had higher service utilization rates in rural areas. Overall, East Azerbaijan, Mazandaran, and Qom provinces had a higher rate of utilization. The $\mathrm{Cl}$ value for the whole
\end{abstract}

\section{${ }^{*}$ Corresponding Author:}

Ali Kazemi Karyani, PhD.

Address: Research Center for Environmental Determinants of Health, Health Institute, Kermanshah University of Medical Sciences, Kermanshah, Iran. Tel: +98 (918) 3558225

E-Mail: alikazemi.k20@gmail.com 
country was reported at 0.24 ( $95 \% \mathrm{Cl}: 0.17-0.30)$, indicating a higher inequality in utilization in favor of the rich. The $\mathrm{Cl}$ values for rural and urban areas were 0.27 and 0.19 , respectively $(P<0.001)$. Conclusion There is inequality in the utilization of rehabilitation services in favor of the rich households in Iran, and low-income households have lower access to these services. The inclusion of rehabilitation services in the primary health insurance package with appropriate pricing and population coverage, and fair distribution of rehabilitation services following the needs of public and private sectors, can increase the access and utilization of rehabilitation services.

\section{Extended Abstract}

\section{Introduction}

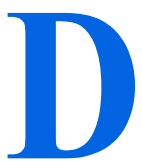

isability has many destructive individual and social effects. According to the "International Classification of Functioning, Disability, and Health", disability is a condition that limits and disrupts a person's participation and activities [1]. The World Health Organization (WHO) estimated that more than one billion people, or $15 \%$ of the world's population, live with some disabilities, $80 \%$ of whom are in low- and middle-income countries [2]. 2017 WHO report showed that about 183 million people have severe disabilities, indicating an increase of $23 \%$ compared to 2005 [3]. People with disabilities, in addition to the need for care from healthy people (such as vaccinations and other prevention and treatment services), need rehabilitation services [4]. Rehabilitation services include a wide range of services that seek to optimize the performance of people with disabilities [2]. The 2015 Global Burden of Diseases study showed that $74 \%$ of all Years Lived with Disabilities (YLDs) in the world was due to disabilities, indicating the need for rehabilitation interventions [3].

Although the demand for rehabilitation services is growing, its supply resources in many parts of the world do not meet the current needs since rehabilitation services have not been considered seriously by many governments [5]. This neglecting has led to improper access to these services and no coordination between the demand and supply [2]. Some studies in South Africa show that only $26 \%$ of the population receives the required rehabilitation services [6-9]. The WHO report in 2014 showed that about $76 \%-85 \%$ of people with disabilities in developing countries do not receive any rehabilitation services [10]. A set of factors influence the utilization of rehabilitation services. To date, various studies have examined the factors affecting the utilization and non-utilization of rehabilitation services [11]. Patel et al. showed that the utilization of rehabilitation services by people with disabilities depends not only on socioeconomic factors but also on cultural factors, residence, literacy status, gender, etc. [12]. Borker et al. showed that the non-utilization of rehabilitation ser- vices by $75.8 \%$ of disabled people in a rural community in India [13]. Another study in 2017 showed that $76.3 \%$ of stroke patients in Ghana did not use rehabilitation services due to economic problems. Some studies have reported low utilization of rehabilitation services by people with disabilities in Iran $[14,15]$. Given the importance and necessity of rehabilitation services, the governments should take the necessary measures to facilitate these services for people in need. To reduce the existing information gap, the present study examines the inequality in utilizing rehabilitation services among Iranian households.

\section{Materials and Methods}

The present study is a descriptive-analytical study with a cross-sectional design using household, expenditure, income survey data from the Statistics Center of Iran in 2018. The relevant data were extracted and categorized by province, urban and rural areas. The initial analysis of raw data was performed. In this regard, the provinces of Sistan and Baluchestan, Ardabil, Kohgiluyeh and Boyer-Ahmad, Kurdistan, and West Azerbaijan were excluded from the study due to lack of data on the use of rehabilitation services (audiometry, optometry, speech therapy, and physiotherapy). Households that declared zero essential expenses (e.g. in food expenses) were also excluded from the study. Finally, the relevant data of 15929 households in rural areas, 17467 households in urban areas, and 38958 households in the whole country were included in the study.

The Chi-square test was used to investigate the relationship between the utilization and non-utilization of rehabilitation services with the study variables. In the next step, inequality in the utilization of rehabilitation services was measured using the Concentration Index (CI). To calculate this index, household income was used as a ranking variable to measure inequality. Households were divided into five categories based on income level from the first quintile (with the lowest income) to the fifth quintile (with the highest income). The CI was calculated as Fourmula 1:

$$
\text { (1) } C I=\frac{2 \times \operatorname{cov}\left(y_{i} r_{i}\right)}{\mu}
$$

where $\mu$ represents the average rate of the dependent variable (percentage of households using rehabilitation 
services), $r_{i}$ refers to the ranking of each household according to the income quintile, and $y_{i}$ shows the utilization of rehabilitation services by household $i$. The numerical value of the CI is between -1 and +1 . The positive value indicates that the use of rehabilitation services is higher among households with higher economic status. The negative value indicates that it is higher among households with lower economic status. In this equation, the dependent variable value is 0 or 1 and is not bounded within the range of -1 and +1 . The normalization of the concentration index was performed by multiplying the value of CI by $1 / 1-\mu$, according to Wagstaff [16].

Access and Excel applications were used for data extraction and STATA V.14.1 software for data analysis. To better describe each province's situation in the utilization of rehabilitation services, the rate of use of these services by urban/ rural areas and the whole country was also displayed on the map using ArcGIS Map V.10 software.

\section{Results}

Of the total study households, about $258(0.77 \%)$ had used rehabilitation services in 2018. Among the households that used rehabilitation services, $226(87 \%)$ had a male head, and $32(13 \%)$ had a female head. Also, $52(21 \%)$ had 1-2 members, 156 (60\%) 3-4 members, and 50 (19\%) had $\geq 5$ members. Besides, 239 (92\%) had insurance coverage, and $19(8 \%)$ had no insurance coverage, and this index had a significant effect on the use of rehabilitation services $(\mathrm{P}<0.05)$. Heads of 173 households $(67 \%)$ who used rehabilitation services were employed. Moreover, the households in the fifth quintile of income (36\%) used rehabilitation services more than other quintiles (Table 1). In

Table 1. Household characteristics and their utilization rate of rehabilitation services

\begin{tabular}{|c|c|c|c|c|c|}
\hline & & \multicolumn{2}{|c|}{ No. (\%) } & \multirow{3}{*}{ Total } & \multirow{3}{*}{$\mathbf{P}^{*}$} \\
\hline \multicolumn{2}{|l|}{ Variables } & \multicolumn{2}{|c|}{ Utilization of Rehabilitation Services } & & \\
\hline & & No & Yes & & \\
\hline \multirow{3}{*}{ Gender of the household head } & Male & 28700 (99.22) & $226(0.78)$ & 28926 & \multirow{3}{*}{0.64} \\
\hline & & & & & \\
\hline & Female & $4438(99.28)$ & $32(0.72)$ & 4470 & \\
\hline \multirow{3}{*}{ Household size } & $1-2$ & $8930(99.42)$ & $52(0.58)$ & 8982 & \multirow{3}{*}{$<0.05$} \\
\hline & $3-4$ & 17865 (99.12) & $156(0.88)$ & 18021 & \\
\hline & $\geq 5$ & $6343(99.22)$ & $50(0.78)$ & 6393 & \\
\hline \multirow{3}{*}{ Insurance coverage } & No & $4007(99.53)$ & $19(0.47)$ & 4026 & \multirow{3}{*}{$<0.05$} \\
\hline & & & & & \\
\hline & Yes & $29131(99.13)$ & $239(0.81)$ & 29370 & \\
\hline \multirow{3}{*}{ Employment of the household head } & No & $10769(99.22)$ & $85(0.78)$ & 10854 & \multirow{3}{*}{0.87} \\
\hline & & & & & \\
\hline & Yes & $22369(99.23)$ & $173(0.77)$ & 22542 & \\
\hline \multirow{3}{*}{ Location } & Rural area & $17318(99.15)$ & $142(0.85)$ & 17467 & \multirow{3}{*}{0.09} \\
\hline & & & & & \\
\hline & Urban area & $15820(99.32)$ & $111(0.62)$ & 17917 & \\
\hline \multirow{5}{*}{ Income quintile } & $1^{\text {st }}$ (Lowest) & 5985 (99.65) & $21(0.35)$ & 6006 & \multirow{5}{*}{$<0.001$} \\
\hline & $2^{\text {nd }}$ & $6696(99.45)$ & $37(0.55)$ & 6733 & \\
\hline & $3^{\text {rd }}$ & $6676(99.24)$ & $51(0.76)$ & 6727 & \\
\hline & $4^{\text {th }}$ & 6850 (99.19) & $56(0.81)$ & 6906 & \\
\hline & $5^{\text {th }}$ (Highest) & 6931 (99.68) & 93 (1.32) & 7024 & \\
\hline Total & & $33138(99.23)$ & $258(0.77)$ & 33396 & - \\
\hline
\end{tabular}

"The Chi-square test. 
Table 2. Statistics related to the utilization of rehabilitation services by Iranian households categorized by the province of residence

\begin{tabular}{|c|c|c|c|}
\hline \multirow{3}{*}{ Province } & \multirow{3}{*}{ Number of Households } & \multicolumn{2}{|c|}{ No. (\%) } \\
\hline & & \multicolumn{2}{|c|}{ Utilization of Rehabilitation Services } \\
\hline & & No & Yes \\
\hline Markazi & 1433 & $1420(99)$ & $13(1)$ \\
\hline Guilan & 1320 & $1311(99.30)$ & $9(0.70)$ \\
\hline Mazandaran & 1036 & $1020(98.46)$ & $16(1.54)$ \\
\hline East Azerbaijan & 1280 & $1263(98.67)$ & $17(1.33)$ \\
\hline Kermanshah & 1373 & $136(99.34)$ & $9(0.66)$ \\
\hline Khuzestan & 1385 & $1378(99.5)$ & $7(0.50)$ \\
\hline Fars & 1494 & $1479(99)$ & $15(1)$ \\
\hline Kerman & 1090 & 1085 (99.54) & $5(0.46)$ \\
\hline Khorasan Razavi & 1607 & 1595(99.32) & $11(0.68)$ \\
\hline Esfahan & 1339 & $1333(99.55)$ & $6(0.45)$ \\
\hline Hamedan & 1375 & $1366(99.35)$ & $9(0.65)$ \\
\hline Chaharmahal va Bakhtiari & 1167 & $1157(99.14)$ & $10(0.86)$ \\
\hline Lorestan & 1044 & $1042(99.62)$ & $4(0.38)$ \\
\hline Ilam & 1008 & $1002(99.40)$ & $6(0.60)$ \\
\hline Bushehr & 1116 & $1113(99.73)$ & $3(0.27)$ \\
\hline Zanjan & 1123 & $1116(99.38)$ & $7(0.62)$ \\
\hline Semnan & 959 & 955 (99.58) & $4(0.42)$ \\
\hline Yazd & 1258 & $1243(98.81)$ & $15(1.19)$ \\
\hline Hormozgan & 1568 & 1554 (99.11) & $14(0.89)$ \\
\hline Tehran & 2020 & 2004 (99.21) & $16(0.79)$ \\
\hline Qom & 929 & 914 (99.39) & $15(1.61)$ \\
\hline Qazvin & 987 & 983 (99.59) & $4(0.41)$ \\
\hline Golestan & 1749 & $1728(98.80)$ & $21(1.20)$ \\
\hline North Khorasan & 1407 & $1398(99.36)$ & $9(0.64)$ \\
\hline Southern Khorasan & 1356 & 1349 (99.48) & $7(0.52)$ \\
\hline Alborz & 971 & 965 (99.38) & $6(0.62)$ \\
\hline $\mathrm{P}$ & & $<0.001$ & \\
\hline
\end{tabular}



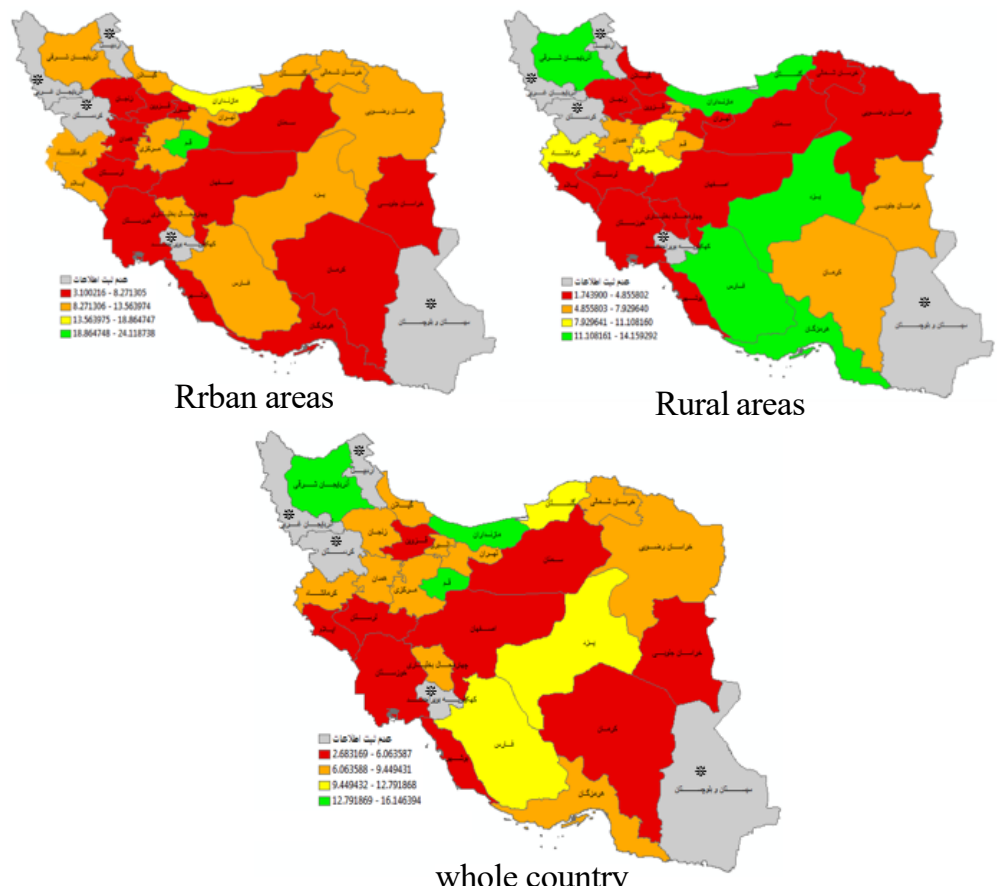

whole country

Archives of

Figure 1. Geographic distribution of the rehabilitation service utilization by Iranian households in urban/rural areas and the whole country in 2018 (per 1000 households)

terms of the utilization at the provincial level, the lowest household rate using rehabilitation services was related to Bushehr Province $(0.27 \%$ ), followed by Lorestan, Semnan, Isfahan, and Kerman. The highest rate was related to Qom, Mazandaran, East Azerbaijan, Golestan, and Yazd provinces $(>1 \%)$ (Table 2$)$.

According to the utilization mapping (Figure 1), Zanjan, Qazvin, Khuzestan, Isfahan, Lorestan, Bushehr, and Semnan provinces had the lowest use of rehabilitation services in urban and rural areas and the whole country (shown in red on the map). In urban areas, only the situation in Qom province was reported to be high. In contrast, a high utilization rate was reported in rural areas of East Azerbaijan, Mazandaran, Golestan, Yazd, Fars, and Hormozgan provinces. In the whole country, only for the provinces of East Azerbaijan, Mazandaran, and Qom, a high level of utilization was reported (shown in green on the map).

The study of inequality in the utilization of rehabilitation services in the whole country showed a concentration index of $\mathrm{CI}=0.24$ (95\% CI: $0.17-0.30)$, indicating inequality in favor of the rich. This condition suggests that the rich use more of these services than the poor. The value of this index for rural and urban areas was 0.27 and 0.19 , respectively $(\mathrm{P}<0.001)$. At the provincial level, the CI values for the provinces of Kermanshah, Kerman, Isfahan, and Ilam were negative, indicating inequality in favor of the poor. However, these values were not significantly different from zero (equality in service utilization). In 11 out of 26 provinces, inequality was statistically significant $(\mathrm{P}<0.001)$. Among these, the highest level of inequality was related to Bushehr $(\mathrm{CI}=0.74)$, Lorestan $(\mathrm{CI}=0.70)$, and North Khorasan $(\mathrm{CI}=0.59)$ provinces. The lowest $\mathrm{CI}$ value was related to Khorasan provinces $(\mathrm{CI}=0.04)$, but it was not statistically significant. The lowest inequality that was statistically significant was related to Qom province $(\mathrm{CI}=0.26)$ (Table 3 ).

\section{Discussion and conclusion}

The purpose of this study was to investigate the distribution of the utilization of rehabilitation services among Iranian households. The results showed that about $0.77 \%$ of the Iranian households in 2018 had used rehabilitation services. Considering the prevalence of disability in Iran in 2011 , which was reported to be about $1.35 \%$, this finding can be justified [17]. The type of population can also affect the use of services. A study by Fullard et al. in the United States found that about $14 \%$ of Parkinson patients in 2007 used services such as physiotherapy, occupational therapy, and speech therapy [18].

The value of the concentration index in this study was 0.24 for the whole country, and it was higher in households with 
Table 3. The concentration index values for the inequality in the utilization of rehabilitation services for provinces, rural/urban areas, and the whole country of Iran

\begin{tabular}{|c|c|c|c|}
\hline Province & Concentration Index & $95 \% \mathrm{Cl}$ & $\mathbf{P}$ \\
\hline Markazi & 0.10 & -0.21 to 0.40 & 0.15 \\
\hline Guilan & 0.45 & 0.08 to 0.82 & 0.01 \\
\hline Mazandaran & 0.12 & -0.16 to 0.39 & 0.33 \\
\hline East Azerbaijan & 0.33 & 0.06 to 0.60 & 0.01 \\
\hline Kermanshah & -0.09 & -0.46 to 0.28 & 0.63 \\
\hline Khuzestan & 0.04 & -0.38 to 0.46 & 0.86 \\
\hline Fars & 0.30 & 0.01 to 0.58 & 0.04 \\
\hline Kerman & -0.15 & -0.64 to 0.38 & 0.54 \\
\hline Khorasan Razavi & 0.35 & 0.01 to 0.68 & 0.04 \\
\hline Esfahan & -0.20 & -0.65 to 0.25 & 0.38 \\
\hline Hamedan & 0.36 & -0.01 to 0.72 & 0.05 \\
\hline Chaharmahal va Bakhtiari & 0.12 & -0.23 to 0.47 & 0.51 \\
\hline Lorestan & 0.70 & 0.14 to 1.25 & 0.01 \\
\hline Ilam & -0.11 & -0.56 to 0.34 & 0.63 \\
\hline Bushehr & 0.74 & 0.10 to 1.38 & 0.02 \\
\hline Zanjan & 0.12 & -0.29 to 0.54 & 0.56 \\
\hline Semnan & 0.68 & 0.13 to 1.23 & 0.01 \\
\hline Yazd & 0.40 & 0.11 to 0.69 & $<0.001$ \\
\hline Hormozgan & 0.07 & -0.22 to 0.37 & 0.63 \\
\hline Tehran & 0.17 & -0.10 to 0.43 & 0.22 \\
\hline Qom & 0.26 & -0.03 to 0.54 & 0.08 \\
\hline Qazvin & 0.42 & -0.13 to 0.97 & 0.13 \\
\hline Golestan & 0.14 & -0.10 to 0.38 & 0.25 \\
\hline North Khorasan & 0.59 & 0.22 to 0.95 & 0.002 \\
\hline Southern Khorasan & 0.10 & -0.31 to 0.52 & 0.62 \\
\hline Alborz & 0.19 & -0.24 to 0.63 & 0.38 \\
\hline Whole country & 0.24 & 0.17 to 0.30 & $<0.001$ \\
\hline Rural areas & 0.27 & 0.18 to 0.36 & $<0.001$ \\
\hline Urban areas & 0.19 & 0.09 to 0.30 & $<0.001$ \\
\hline
\end{tabular}


higher income. A study by Ahmadi et al. showed that, for specialized medical and dental services, inequality was in favor of the rich, while for the general medical, family physician, and primary health care services, inequality was in favor of the poor [19]. The study by Rezapour et al. in Kerman also showed that inequality in the use of outpatient and inpatient services was in favor of poorer groups [20].

Results reported that household size and health insurance coverage had a significant relationship with the utilization of rehabilitation services. With the increase of the household size, the use of rehabilitation services increased. Lack of insurance coverage for some rehabilitation services (speech therapy, occupational therapy, and technical orthopedics), long duration of use of these services, and high deductible for services such as physiotherapy led to the higher utilization of these services by the households with higher income. In other words, the use of rehabilitation services had disproportionately been concentrated on high-income households. These results highlight the need to review and modify the basic health insurance package in Iran. Some studies believe that misunderstandings of the provisions of the Public Health Insurance Act by policymakers in the past have led to the exclusion of rehabilitation services from basic health insurance packages [21]. Since studies have shown a higher prevalence of disability among poorer groups in society, it is necessary to make improvements in the regulations of health insurance to increase the benefit of these people from rehabilitation services so that they can receive the rehabilitation services according to their needs and without financial pressure [22-24].

In the present study, Bushehr and Qom provinces of Iran had the highest and lowest inequality in the use of rehabilitation services, respectively, indicating that the income gap of households in Bushehr Province had caused more inequality in the use of rehabilitation services compared to Qom Province. According to the results, inequality in the utilization of rehabilitation services was higher in rural households than in urban households. This condition probably indicates that, due to the higher income gap between households, unfair distribution of rehabilitation services, and low geographical access to these services, lower-income households in less developed areas are less likely to use rehabilitation services. Chavehpour et al. showed that the development rate is directly related to the concentration of health resources such that $70.6 \%$ of hospital beds in Isfahan and Tehran provinces were located in areas with higher social and economic status [25].

Among the provinces, Qom and Bushehr had the highest and lowest rate of using rehabilitation services, respectively. This condition indicates that the share of the public health centers in providing rehabilitation services in Bushehr Province is less than that of the private health centers. Since the cost of these services in private health centers is higher, lower-income groups are less tended to use these services. Other reasons can be the insufficient supply of rehabilitation services or less awareness of these services in Bushehr Province. Vamaghi et al. in a study in Tehran, showed that one of the reasons for not using speech therapy services was the lack of awareness of the parents about the existence of such services [26]. Studies by Rais Dana et al. [53], Soltani et al. [54], and Abdi et al. [7] showed that the existence of cultural factors such as wrong perceptions and attitudes towards people with disabilities could be one of the barriers in using these services in Iran.

The main limitation of the present study was the assumption that households would spend on rehabilitation services. In other words, only households that stated that they had paid for rehabilitation services were considered as households using rehabilitation services. Accordingly, the households that might have received free rehabilitation services for having supplementary insurance were considered as households with no utilization of rehabilitation services. However, studies in Tehran have shown that rehabilitation services covered by insurance are less popular than other services [29], which may be due to the limited options and low quality of available services [30].

Income inequality in the use of rehabilitation services significantly reduces the access of low-income people to these services, including rehabilitation services in the primary health insurance package with appropriate price and population coverage can increase equity in access to rehabilitation services. Moreover, fair distribution of rehabilitation services in accordance with the needs of both public and private rehabilitation centers can play an essential role in increasing the use of these services by households. The committed participation of institutions and organizations such as the Ministry of Health and Medical Education, health insurance organizations, welfare organizations, and Non-Governmental Organizations (NGOs) related to people with disabilities in health policy processes can play an essential role in fulfilling the expectations of this group of people about receiving rehabilitation services.

\section{Ethical Considerations}

\section{Compliance with ethical guidelines}

This study was approved by the Ethics Committee of Kermanshah University of Medical Sciences (Code: IR.KUMS.REC.1398.516). 
Funding

This research was funded by Kermanshah University of Medical Sciences.

Authors' contributions

Study design: Jafar Yahyavi Dizaj, Farogh Nomani, Ali Kazemi Karyani; Methods of study and statistical analysis: Manijeh Soleimanifar, Mohsen Fateh, Shahin Soltani; Data analysis and interpretation: Amir Massoud Arab, Jafar Yahyavi Dizaj, Ali Kazemi, Shahin Soltani; Consulting, editing and final writing of the article: Manijeh Soleimanifar, Farogh Nomani. All authors approve the content article.

Conflict of interest

there is no Conflict of interests.

Acknowledgments

We would like to thank the Statistics Center of Iran for making the data available, as well as the professors who helped guide this study. Kermanshah University of Medical Sciences is also appreciated for its financial support of this research. 
This Page Intentionally Left Blank 


\title{
تأثير نابرابرى درآمدى در استفاده از خدمات توانبخشى خانوارهاى شهرى و روستايى ايران: يكى مطالعهمقطعى
}

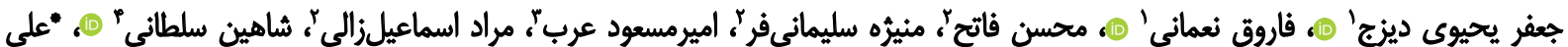

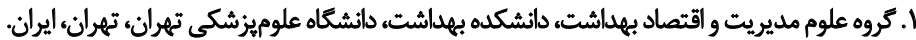

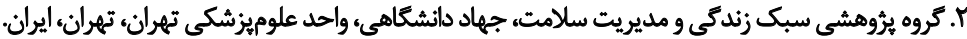

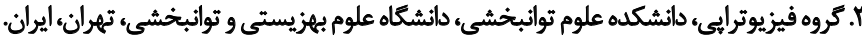

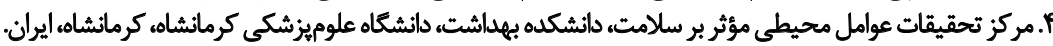

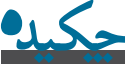

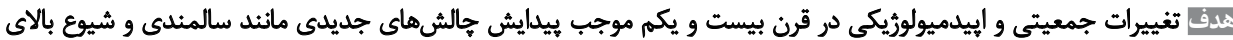

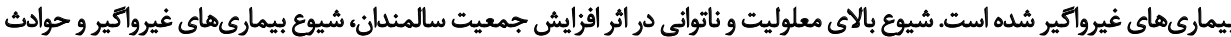

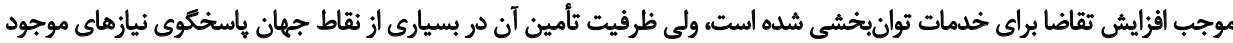

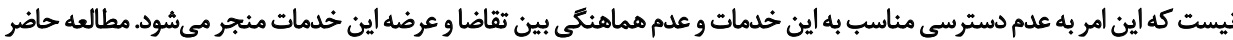

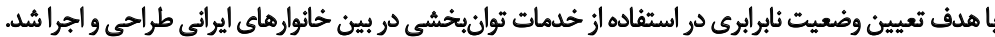

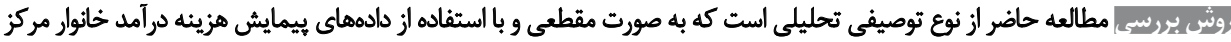

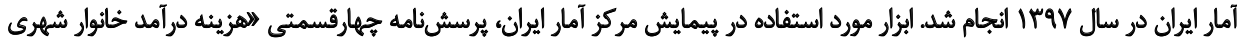

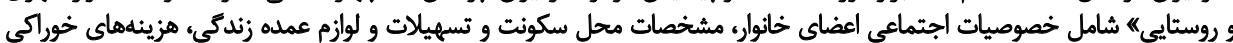

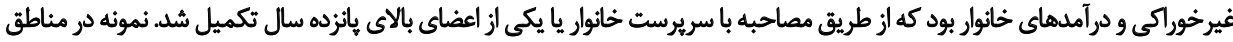

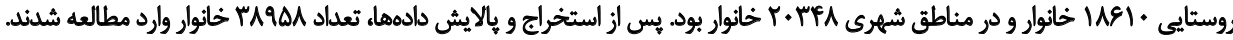

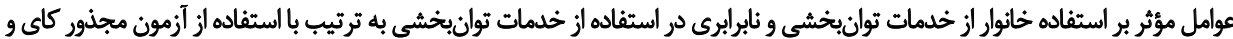

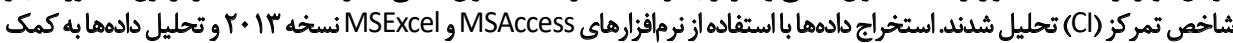

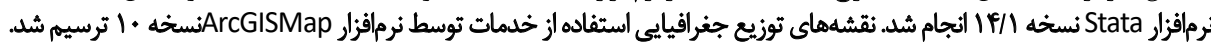

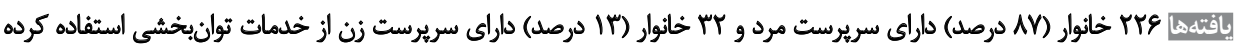

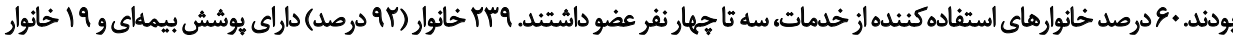

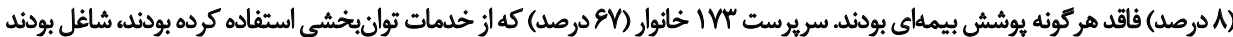

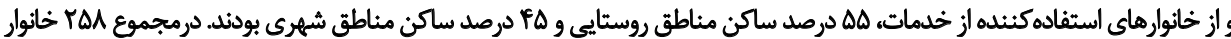

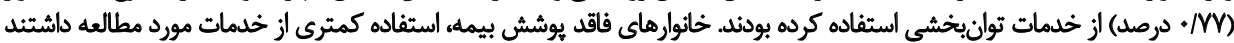

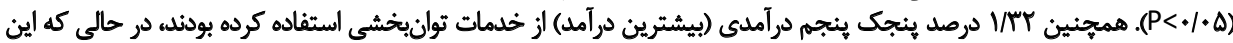

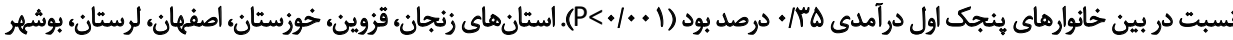

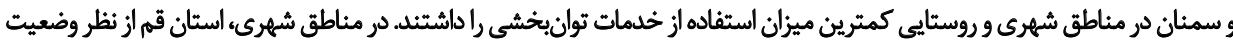

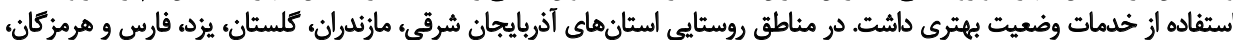

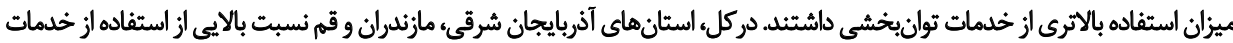

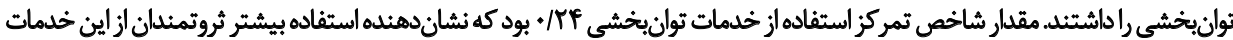

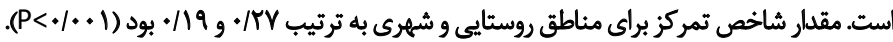

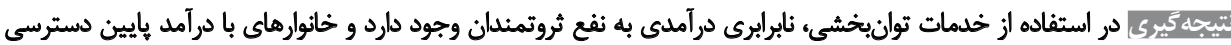

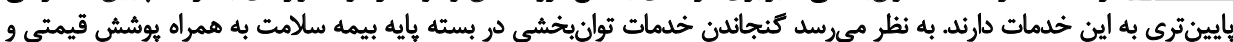

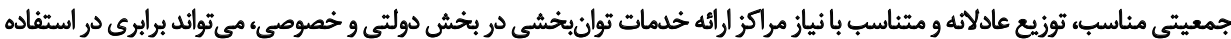

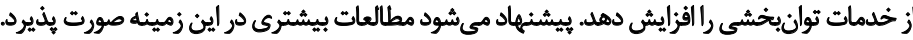

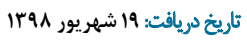

تاريخ هذيرش: 19 بهمن

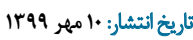

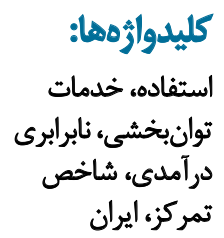

$$
\begin{aligned}
& \text { "نويسنده مسئول: } \\
& \text { دكثر على كاظمى كريانى }
\end{aligned}
$$

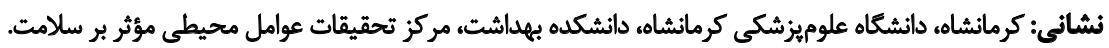

$$
\begin{aligned}
& \text { تلفي: } \\
& \text { رايانامه: alikazemi.k20@gmail.com }
\end{aligned}
$$




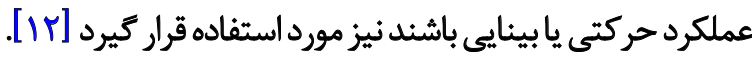
مقدمه

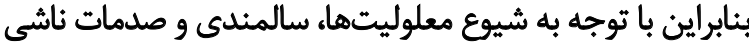

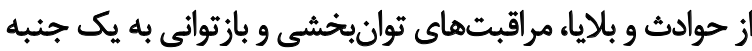

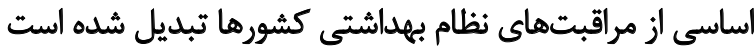

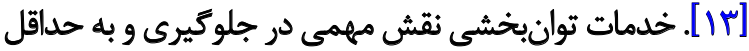

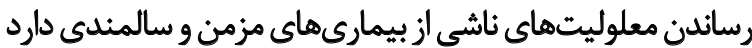

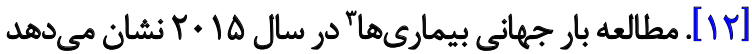

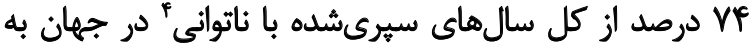

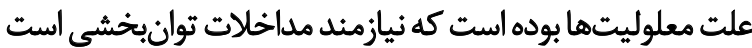

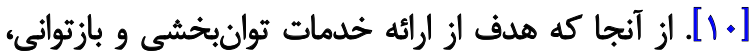

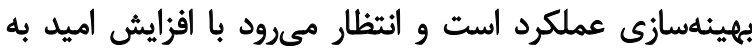

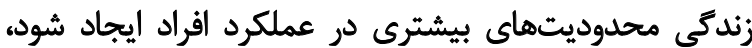

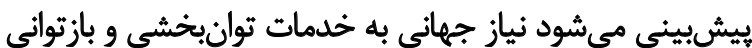

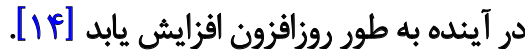

خدمات توانبخشى به خصوص در شرايطى كه فشرده و كاملاً

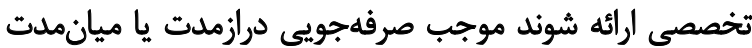

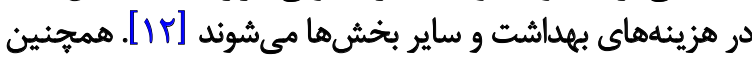

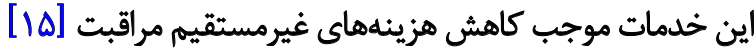

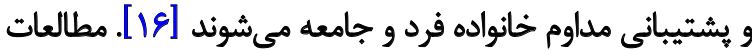

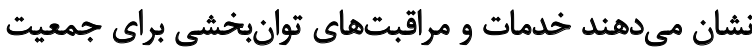

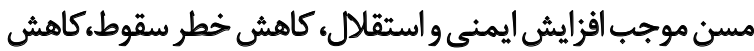

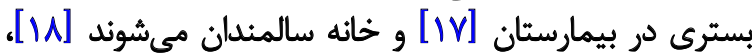

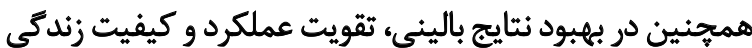

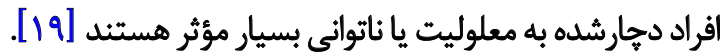

با اينكه تقاضا براى خدمات توانبخشى رو به رشد است، ولى باري

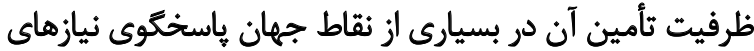

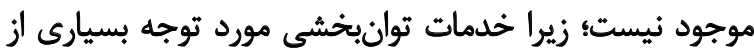

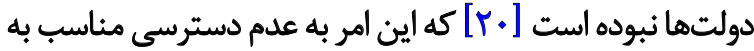

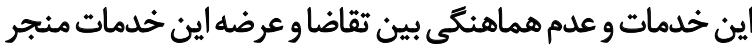

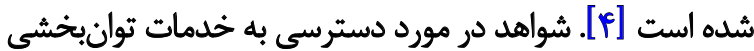

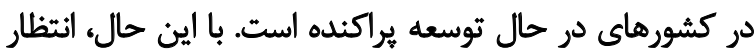

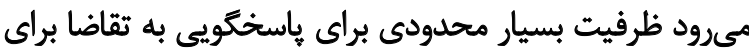

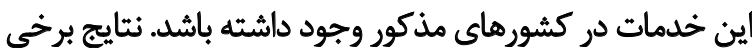

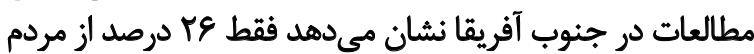

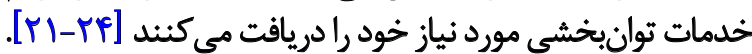

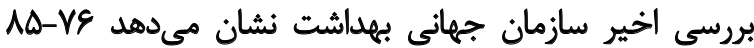

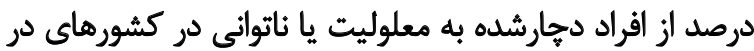

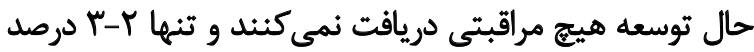

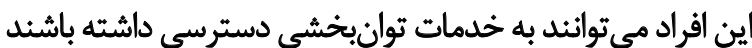

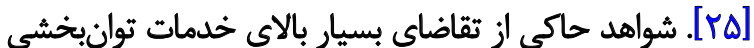

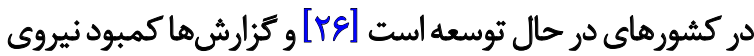

هركاه از سلامتى صحبت مي شوده، اولين و ابتداييترين

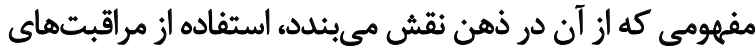

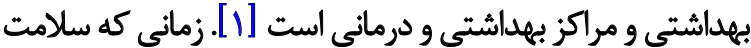

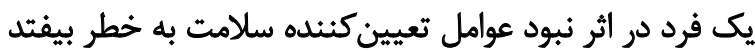

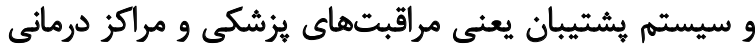

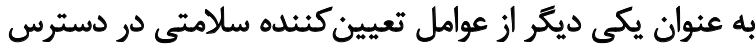

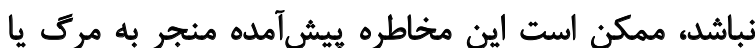

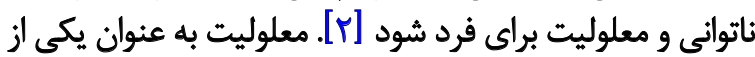

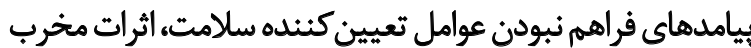

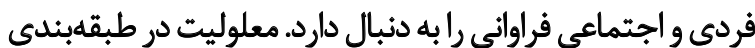

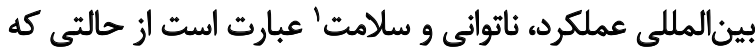

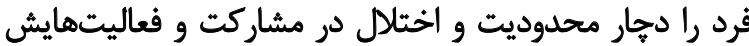

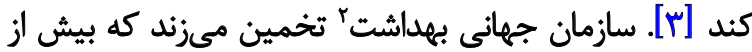

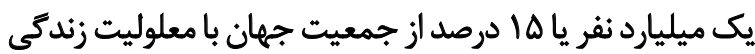

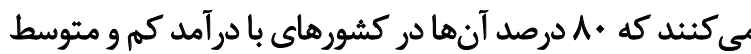

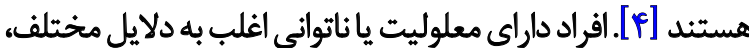

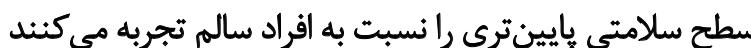

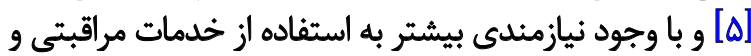

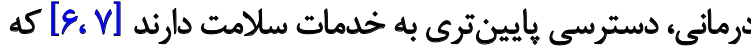

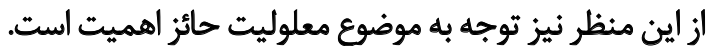

تغييرات جمعيتى و اييدميولوريكى در قرن بيست و يكمه جهان

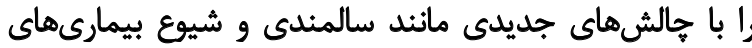

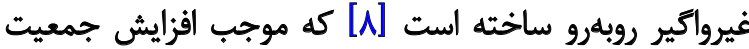

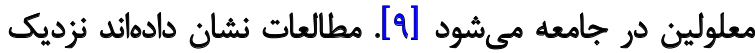

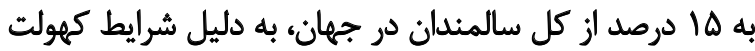

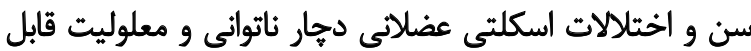

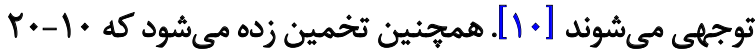

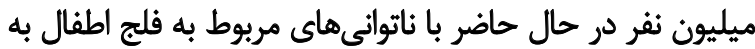

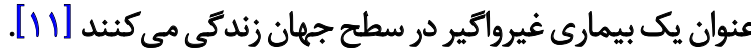

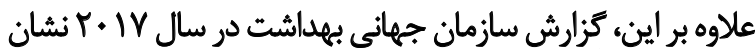

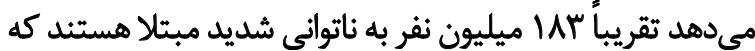

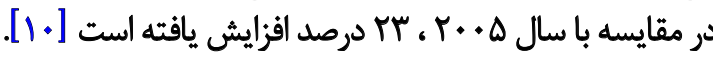
افراد معلول يا ناثوان علاوه بر نياز به مراقبتهاي افراد سالم

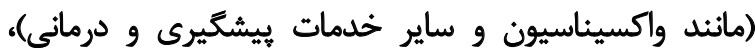

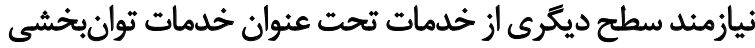

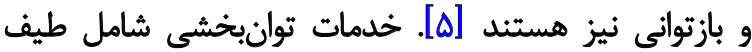

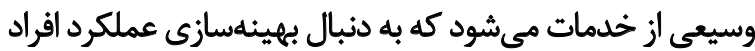

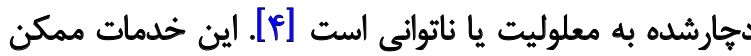

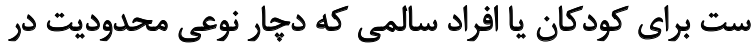


بايد تدابير لازم جهت تسهيل استفاده افراد و خانوارهاى نيازمند

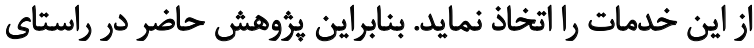

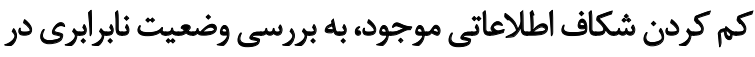

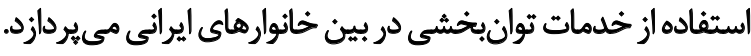

\section{ووش بروسي}

مطالعه حاضر از نوع توصيفى تحليلى، به صورت مقطعى و

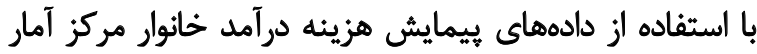

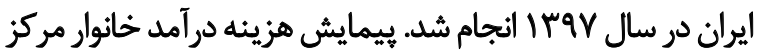

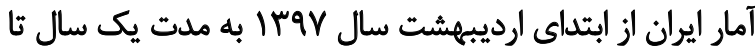

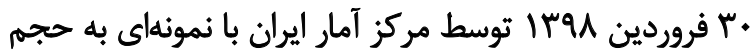

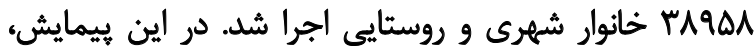

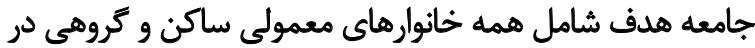
مناطق شهرى و روستايى كشور بود. نمونه

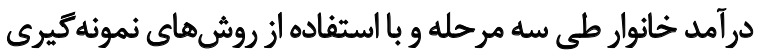

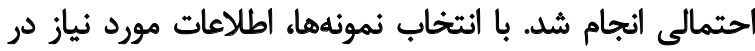

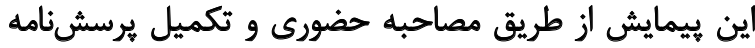

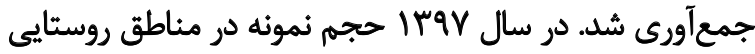

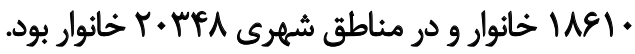

ابزار مورد استفاده در ييمايش مركز آمار ايران، يرسشنامه

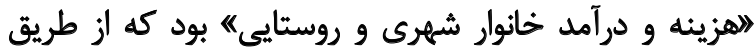

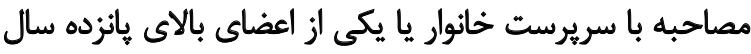

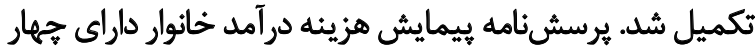

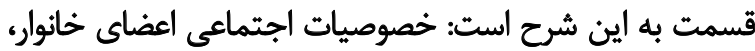

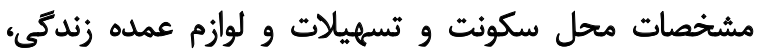
هزينههاى خوراكى و غيرخوراكى و و درآمدهات

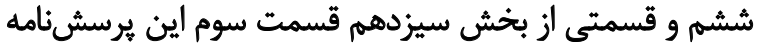

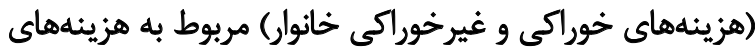

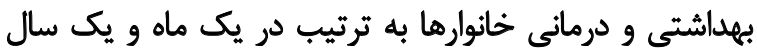

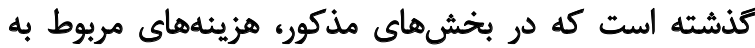

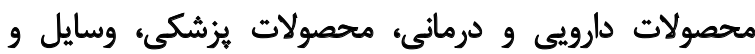

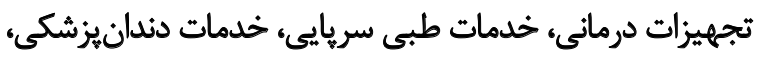

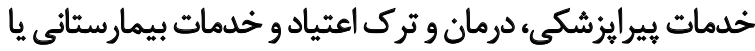

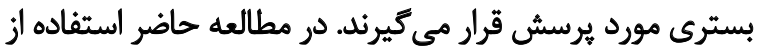

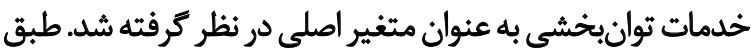

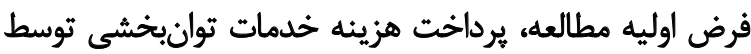

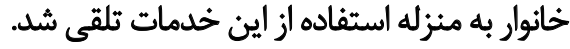

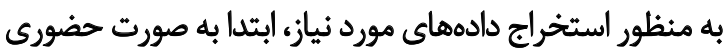

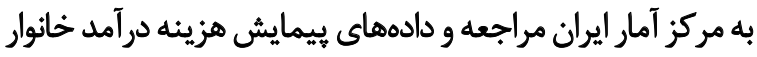

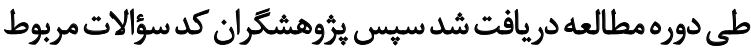

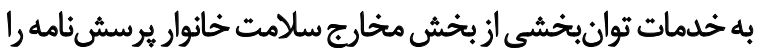

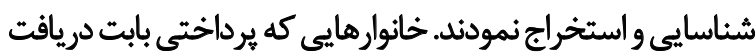
اين خدمات داشتند به عنوان خانوارهاي استفادهكنينده از خدانيات
انساني در حوزههاى حُدمات توانبخشى در اين كشورها رانشان

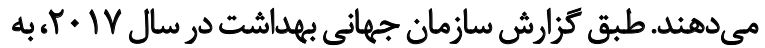

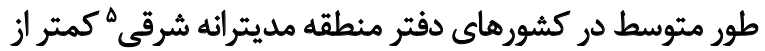

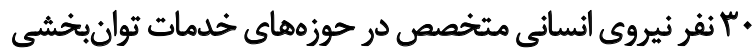

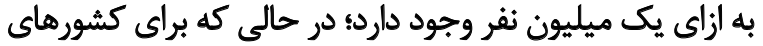

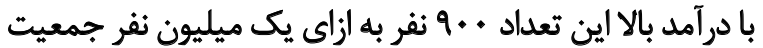

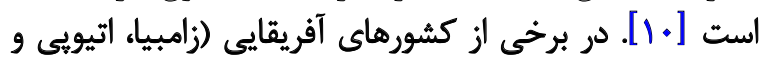

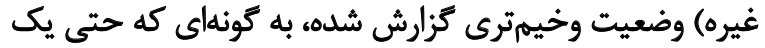

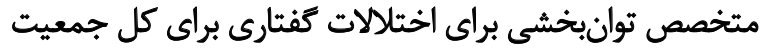

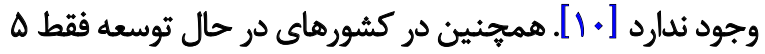

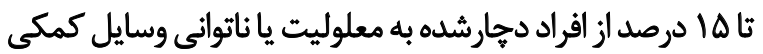
و خدمات مورد نياز خود را دريافت مي كنيند [F]

استفاده ازخدمات توانبخشى تحت تأثير مجموعهاي از عوامل

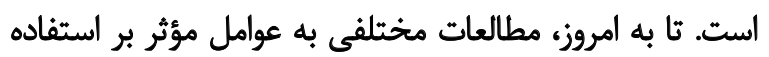

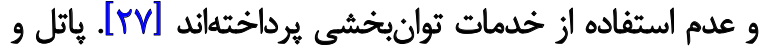

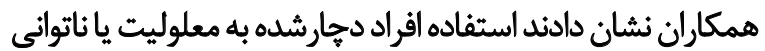

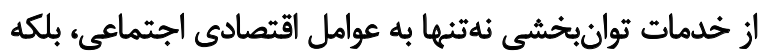

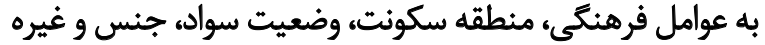

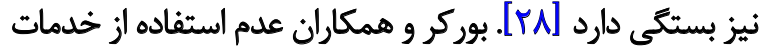

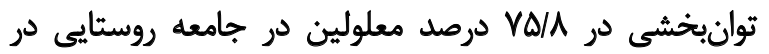

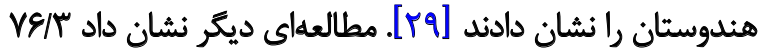

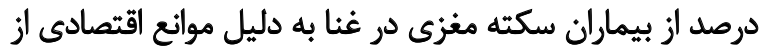

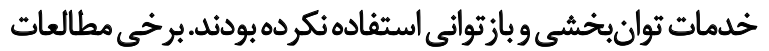

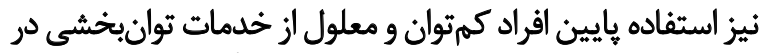

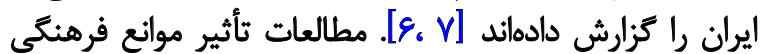

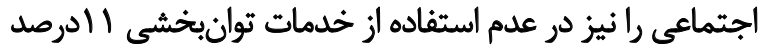

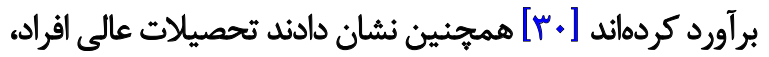

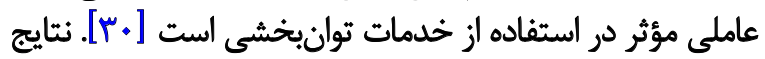

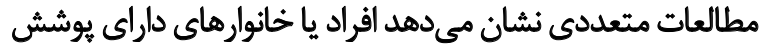

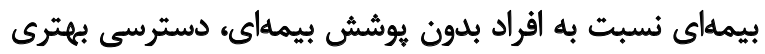

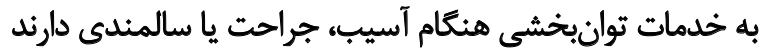

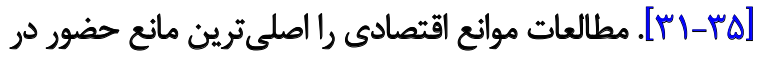

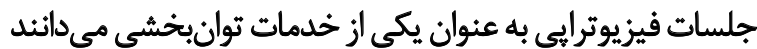

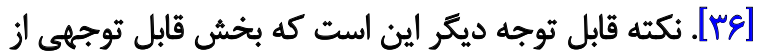

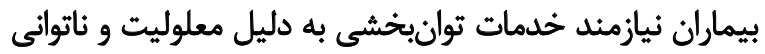

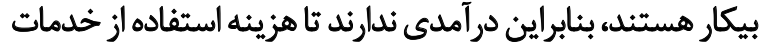

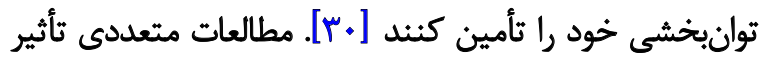

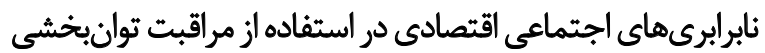

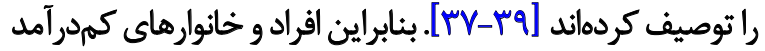

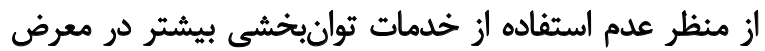

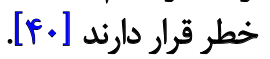
با توجه به اهميت و ضرورى بودن خدمات توانبخشى، دولت

5. Eastern Mediterranean Regional Office (EMRO) 
جدول ا. توزيع وضعيت استفاده از خدمات توانبخشى در خانوارهاى ايرانى به تفكيك مشخصات خائوار و نتايج آزمون مجذور كاى آيا خانوار از خدمات توانبخشى استفاده كرده استى

\begin{tabular}{|c|c|c|c|c|c|}
\hline \multirow{3}{*}{ سطح معنى دارى } & \multirow{3}{*}{ جمع كل } & \multicolumn{2}{|c|}{ آيا خانوار از خدمات توانبخشى استفاده كرده است؟ } & \multirow{2}{*}{\multicolumn{2}{|c|}{ مثغيرها }} \\
\hline & & \multicolumn{2}{|c|}{ تعداد (درصد) } & & \\
\hline & & بله & حير & & \\
\hline \multirow{3}{*}{. $/ 9 t$} & rAqTE & $\operatorname{TrE}(\cdot / \mathrm{NA})$ & YAY.. (9QRTY) & مرد & \multirow{3}{*}{ جنسيت سرير ست خانوار } \\
\hline & & & & & \\
\hline & miv. & $r T(\cdot M)$ & $\operatorname{TTMA}(99 / T \Lambda)$ & زن & \\
\hline \multirow{3}{*}{$<+1 \cdot \Delta$} & Aqur & $\operatorname{Qr}(\cdot / \Delta A)$ & А१พ. (99/\%T) & يك ثادو نفر & \multirow{3}{*}{ 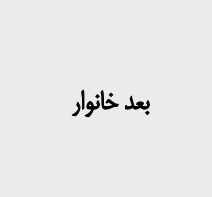 } \\
\hline & $|A \cdot r|$ & $1 \Delta \%(\cdot / M)$ & IVAEs (१Q/IT) & سله تا جهار نفر & \\
\hline & बrat & $0 \cdot(\cdot / V A)$ & बחז (99/TY) & ينج نفر و ييشتر & \\
\hline \multirow[b]{2}{*}{$<+1+\Delta$} & $r \cdot Y \varepsilon$ & $19(. / 19)$ & $P \bullet r(9 Q / \Delta Y)$ & خير & \multirow[b]{2}{*}{ يوشش بيمه } \\
\hline & rapr. & Mra $(\cdot|A|)$ & หqाr) (qด/Ir) & بلى بلى & \\
\hline \multirow[b]{2}{*}{ IAV } & $1+1 A F$ & $\Delta \Delta(\cdot / \mathrm{NA})$ & 1.Vรq (99/TY) & خير & \multirow[b]{2}{*}{ ا اشتغال سريرست خانوار } \\
\hline & TMATT & $\ln (\cdot / m)$ & rmsq (99/Tा) & بلى & \\
\hline \multirow[b]{2}{*}{.1 .9} & iner & $\operatorname{IFr}(\cdot / 1 \Delta)$ & $\ln \mid 1 /(99 / 10)$ & روستا & \multirow[b]{2}{*}{ محل سكونت } \\
\hline & ivily & $111(\cdot 185)$ & $1 Q A Y \cdot(99 / M T)$ & شيهر & \\
\hline \multirow{5}{*}{$<\bullet / \bullet 1$} & $9 . .8$ & $M(\cdot / r \Delta)$ & 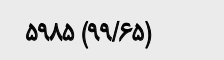 & اول (ققيرترين) & \multirow{5}{*}{ ئنجى درآمد } \\
\hline & ent & $\operatorname{TV}(\cdot / \Delta \Delta)$ & $\operatorname{egqe}(99 / 4 \Delta)$ & دوم & \\
\hline & ent & $\Delta 1(\cdot / V E)$ & exve (99/T) & سوم & \\
\hline & 99.8 & $\Delta \&(\cdot / 1))$ & $8 N \Delta \cdot(99 / 19)$ & جهارم & \\
\hline & $V \cdot r f$ & qr $(1 / M)$ & EATI (99/81) & هنجم+(ثروتمندترين) & \\
\hline- & & $\operatorname{ran}(\cdot M)$ & 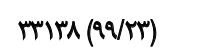 & & \\
\hline
\end{tabular}

همجنين به منظور تعيين عوامل مؤثر بر استفاده خانوار از

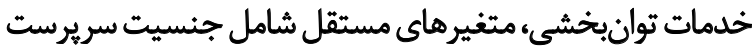

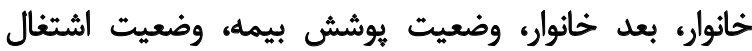

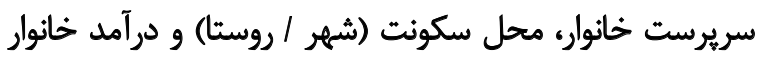

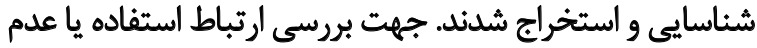

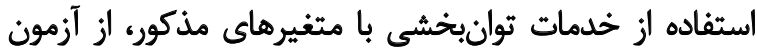

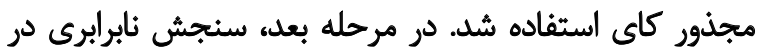

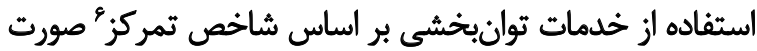

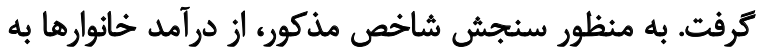

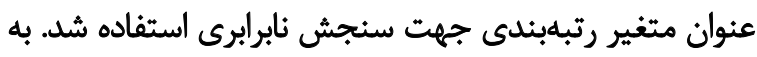

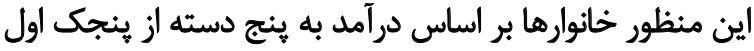

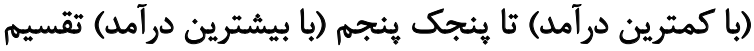

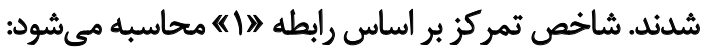

توانبخشى نشان كذارى شدند وخانوارهايى كه يرداختى براى اين

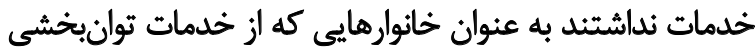

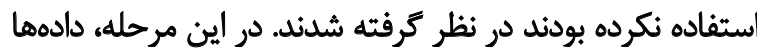

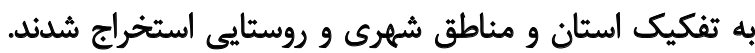

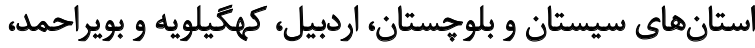

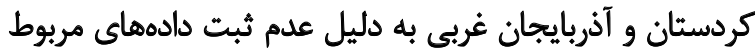

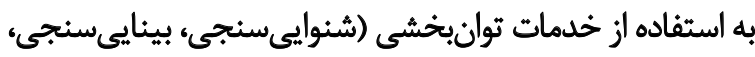

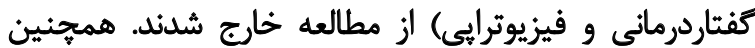

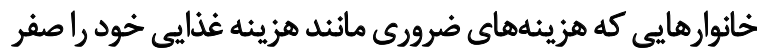

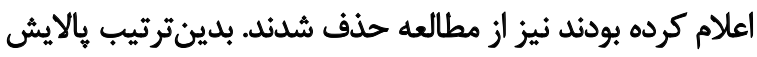

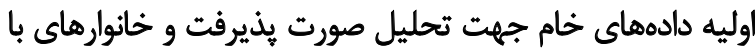

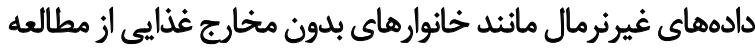

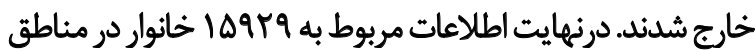

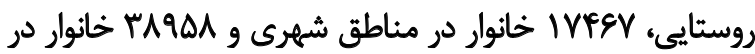
كل كشور مورد بررسى قرار كرفتيند. 
جدول †. نسبت استفاده از خدمات توانبخشى در خانوارهاي ايرانى بر حسب استان محل سكونت آيا خانوار از خدمات توانبخشى استفاده كرده است؟

\begin{tabular}{|c|c|c|c|}
\hline \multicolumn{2}{|c|}{ تعداد خانوار (درصد) } & \multirow[t]{2}{*}{ مجموع خانوارها } & \multirow[t]{2}{*}{ استان } \\
\hline بلي & خير & & \\
\hline $\mathbb{W}(1)$ & IFT. (99) & InT & هركزى \\
\hline $9(\cdot / v \cdot)$ & $|m| \mid(99 / \%+)$ & ITr. & كيالن \\
\hline$\left.I E(N / \Delta)^{\circ}\right)$ & 1.T. (WVeE) & $1 . \mathrm{He}$ & مازنثلران \\
\hline $\operatorname{Ir}(1 / \pi T)$ & ITET (WNEV) & IrA. & آذربايجان شرقى \\
\hline $9(.189)$ & Irefe (99/Me) & irn & كرعانشاه \\
\hline$V(\cdot / \Delta \cdot)$ & $\operatorname{IrVA}(99 / \Delta)$ & irns & خوزستان \\
\hline $18(1)$ & Ifra (99) & ifqF & فارس \\
\hline$\Delta(. / \%)$ & $1 \cdot 1 \Delta\left(9 \vee \Delta \varphi^{\circ}\right)$ & 1.9. & كرمان \\
\hline $11(.18 \mathrm{~A})$ & $1098(99 / \pi T)$ & $18 \cdot r$ & خراسان رضوى \\
\hline $9(. / F \Delta)$ & 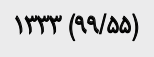 & Irqq & اصفهان \\
\hline $9(. / 90)$ & $1+E g(9 q / r \Delta)$ & irva & همدان \\
\hline $1 \cdot(\cdot / N)$ & $11 \Delta V(99 / 1 F)$ & $119 \mathrm{~V}$ & جهارمحال وبختيارى \\
\hline$P(\cdot / r \Lambda)$ & I.Pr (9V/R) & $1 .+4$ & ل لرستان \\
\hline $8(.18)$. & $1 \ldots r(99 / 4 \cdot)$ & 1.01 & إيلام \\
\hline$r(\cdot / r V)$ & $111 \%(99 / 17)$ & 1118 & يوشهر \\
\hline$V(. / A T)$ & $1118(99 / / \wedge)$ & $11 \pi r$ & زنجان \\
\hline$P(. / F T)$ & $9 \Delta \Delta(99 / \Delta \Lambda)$ & $9 \Delta 9$ & سمنان \\
\hline $10(1 / 19)$ & ITPT (WAI) & ITAA & يزد \\
\hline If $(\cdot / 19)$ & 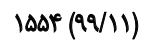 & $\mid A F A$ & هرمزكان \\
\hline $18(\cdot / 19)$ & $r++r(9 q / r))$ & $r \cdot r$. & تمهران \\
\hline $10(1 / 81)$ & $91 \%(99 / 79)$ & १५ & قمم \\
\hline$P(\cdot / F)$ & $R r(99 / \Delta q)$ & ur & ق قزوين \\
\hline$M(N / T \cdot)$ & $\operatorname{IVA}\left(W A_{0}\right)$ & ineq & كلمسان \\
\hline $9(.194)$ & IFq (99/48) & $I f \cdot V$ & خراسان شمالى \\
\hline$Y(\cdot / \Delta T)$ & $\operatorname{lneq}(99 / \% \Lambda)$ & WHAC & خراسان جنوبي \\
\hline $8(. / 8 T)$ & $\vartheta \& \Delta(99 / \mu \mathrm{A})$ & qท & 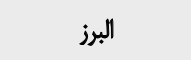 \\
\hline & $<* / \cdot+1$ & & سطح معنى دارى \\
\hline
\end{tabular}




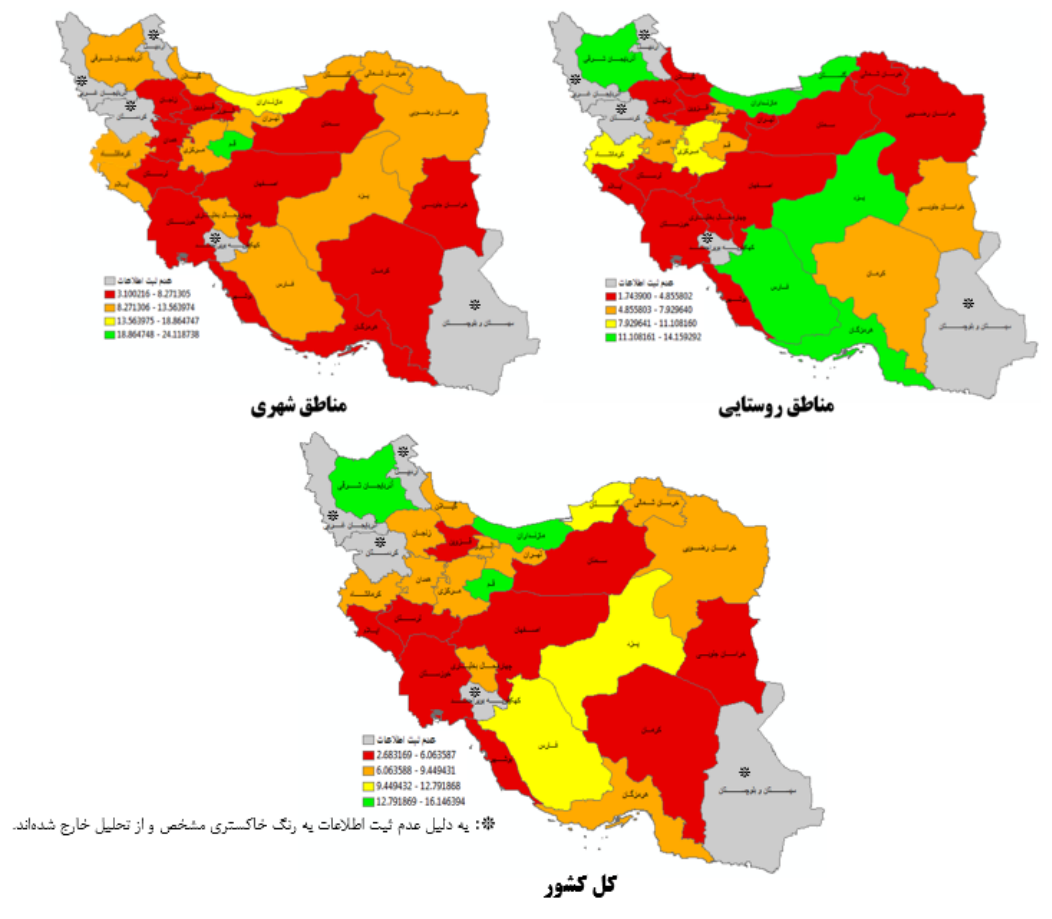

توانبخننى

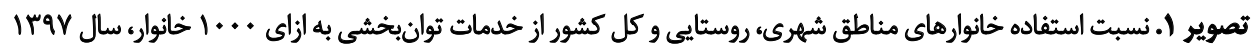

يافتهها

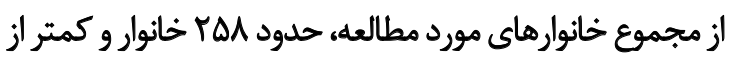

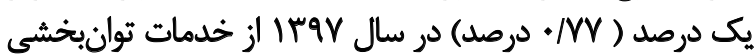

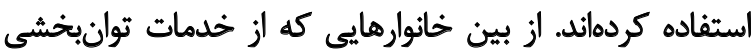

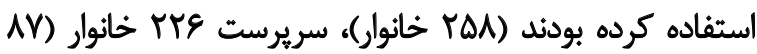

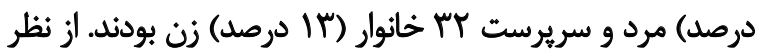

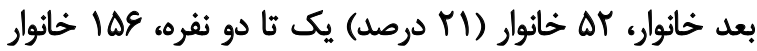

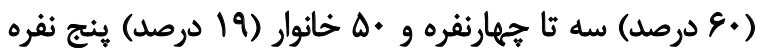

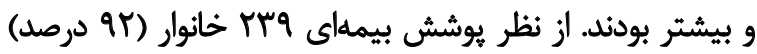

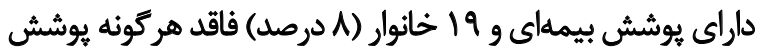

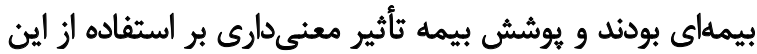

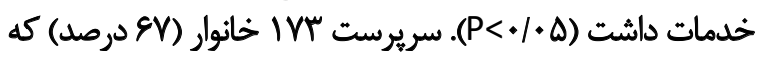

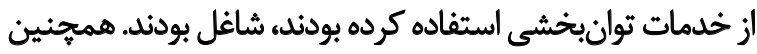

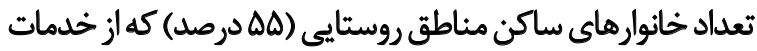

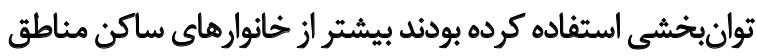

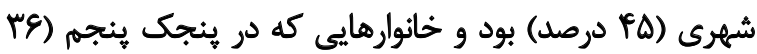

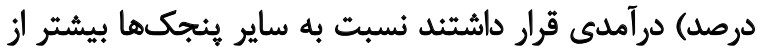

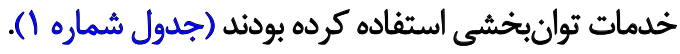

از نظر توزيع استفاده خانوارها از خدمات توانبخشى در سطح

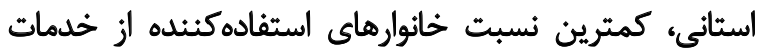

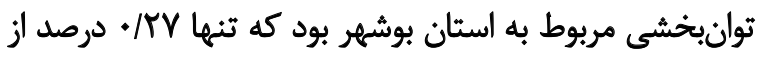

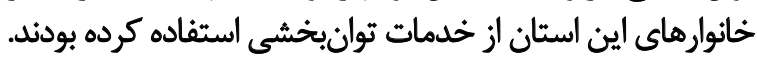
بعد از اين استان، استانهاى لرستان، سمنان، اصفهان و كرمان

$$
C I=\frac{2 \times \operatorname{cov}\left(y_{i}\right.}{\mu} \text { رابطه }
$$

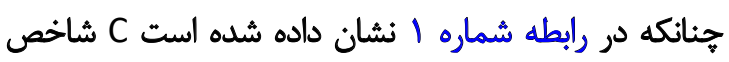

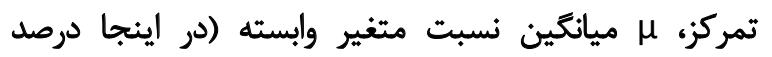

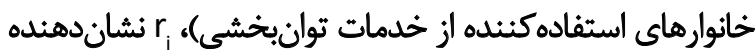

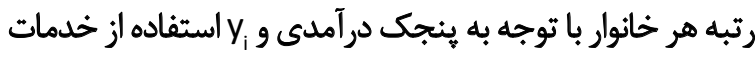

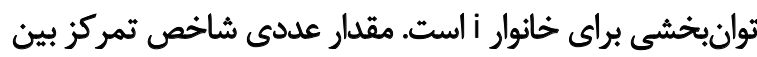

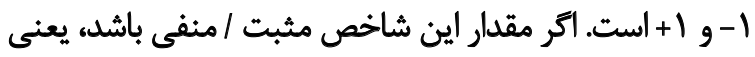

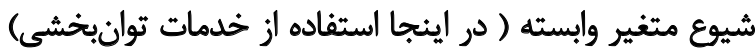

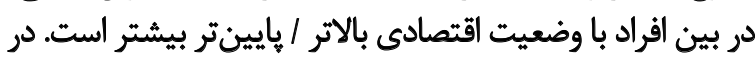

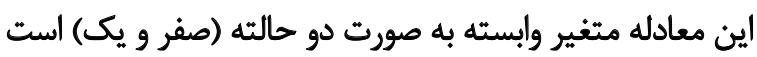

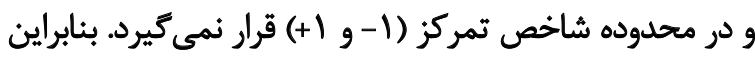

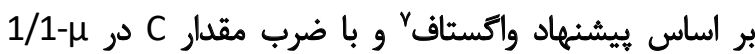

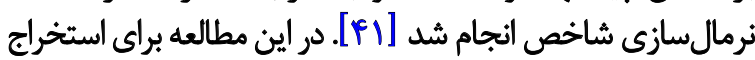

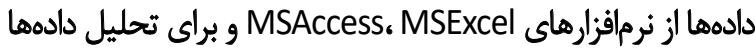

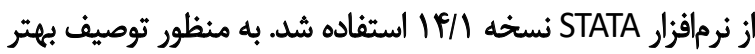

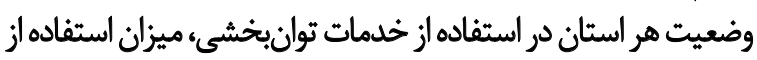

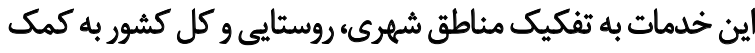

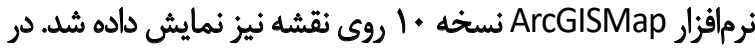

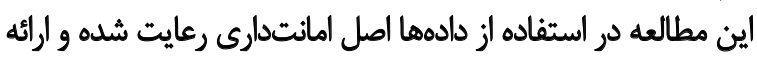

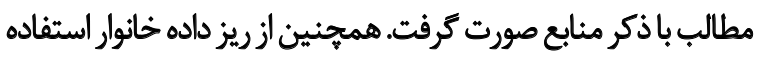

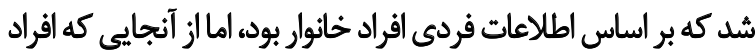

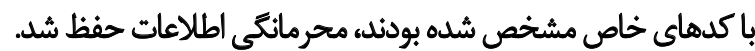


جدول ب. شاخص تمركز استفاده از خدمات توانبخشى در ايران بر حسب استان و مناطق شهرى و روستايى در سال لوجا

\begin{tabular}{|c|c|c|c|}
\hline 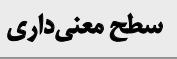 & قاصله اطمينان هو درصد & شاخُص تمركز (C) & اسيتان \\
\hline$\cdot / 1 \Delta$ & $\cdot|f \cdot|-\cdot|r|$ & $\cdot /$ & 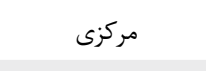 \\
\hline $.1 \cdot 1$ & 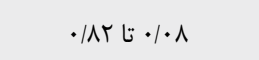 & $\cdot / \& \Delta$ & كيلان \\
\hline אז/. & ع &.$/ 1 Y$ & مازندران \\
\hline $.1 \cdot 1$ & | & אז/. & آذربايجان شرقى \\
\hline .94 & 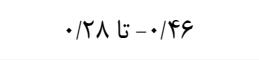 &.$- \cdot 1 \cdot 9$ & كرمانشاه \\
\hline$\cdot / 19$ & لي & $\cdot 1 \cdot r$ & خوزستان \\
\hline$\cdot / \cdot f$ & | & $\cdot / r$ & 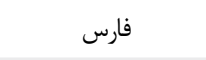 \\
\hline$\cdot / \Delta F$ & 更 & $-\cdot / 10$ & كرمان \\
\hline$\cdot / \cdot f$ & | & $\cdot / \pi \Delta$ & خراسان رضوى \\
\hline$\cdot \pi \wedge$ & 的 • & $-\cdot / r$ & اصفهان \\
\hline$\cdot / \cdot \Delta$ & 1 & 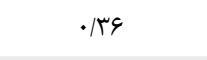 & همدان \\
\hline$\cdot|\Delta|$ & rr/ & ./IT & جهارمحال و بختيارى \\
\hline$\cdot / \cdot 1$ & I/rat & $\cdot / v$ & لرستان \\
\hline . ( ) & 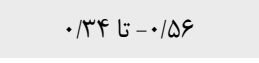 & $-\cdot / 11$ & ايلام \\
\hline$\cdot / \cdot r$ & • & $\cdot / V F$ & بوشهر \\
\hline$\cdot \mid \Delta \varphi$ & 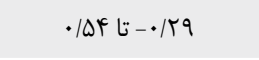 &.$/ 14$ & 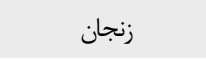 \\
\hline.$/ \cdot 1$ & سו/• تا سז/ & $\cdot 19 \Lambda$ & سمنان \\
\hline$<\cdot / \cdot \cdot 1$ & 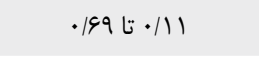 & $\cdot / 4$ & 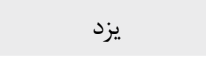 \\
\hline 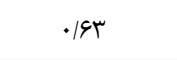 & 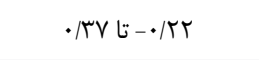 & $\cdot / \cdot v$ & هرمزكان \\
\hline.$/ 4 t$ & • 1/.-تاسع|. & $\cdot / / V$ & 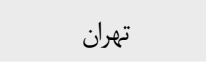 \\
\hline$\cdot 1 \cdot 1$ & r.|• & $\cdot / K \varepsilon$ & قم \\
\hline rו/. & س & . KT & قزوين \\
\hline$\cdot / r \Delta$ & • & $\cdot / 1 F$ & 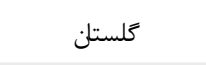 \\
\hline 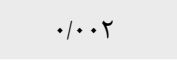 & ז & $\cdot 109$ & خراسان شمالى \\
\hline .94 & | اس/•- تا & $\cdot /$ & خراسان جنوبى \\
\hline ג & F &.$/ 19$ & البرز \\
\hline$<\cdot|\cdot \cdot|$ & • تا • I / V & $\cdot / T F$ & ايران \\
\hline$<\cdot / \cdot \cdot \mid$ & ג//• تاعس/. & $\cdot / T V$ & مناطقروستايى \\
\hline$<\cdot|\cdot \cdot|$ & q•|• ت • • & $\cdot / 19$ & مناطق شهرى \\
\hline
\end{tabular}




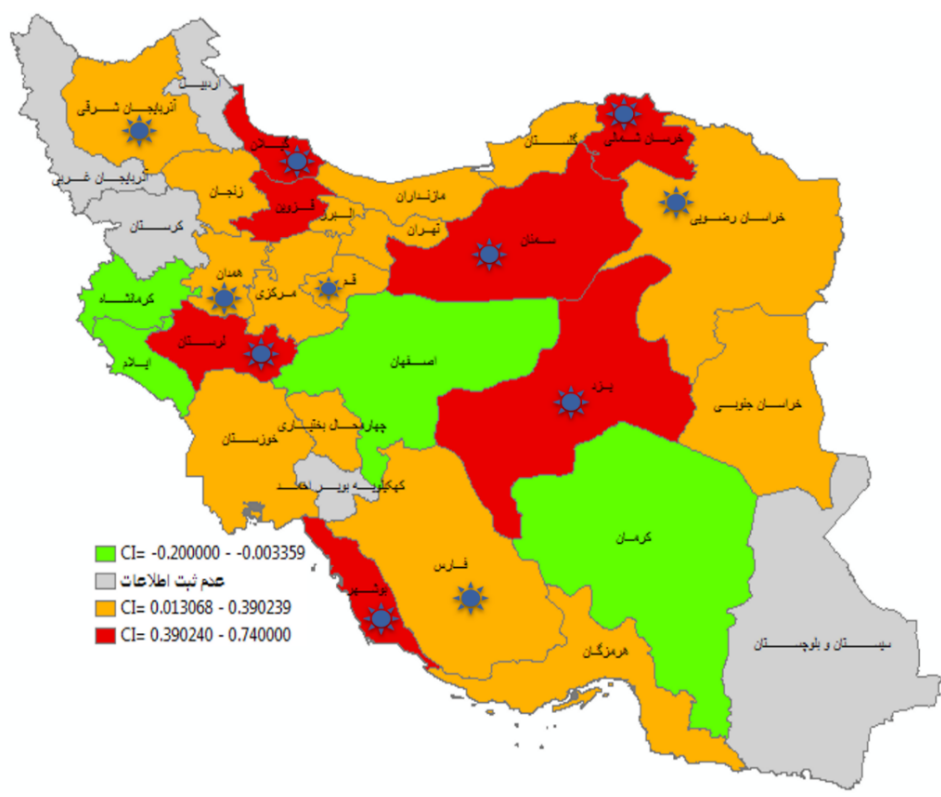

در يازده استان از \& استان مورد مطالعه، نابرابرى از نظر آمارى

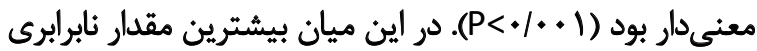

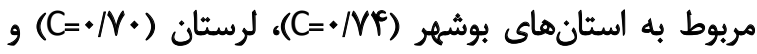

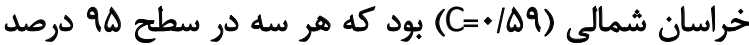

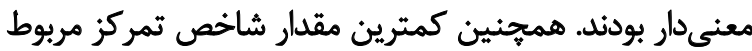

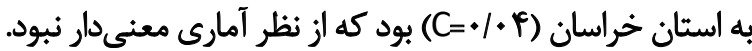

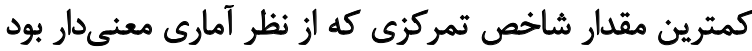

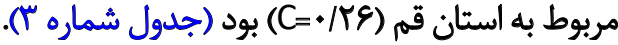
تصوير شماره Y توصيف تصويرى وضعيت نابرابرى درآمدى در

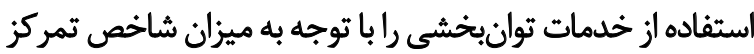

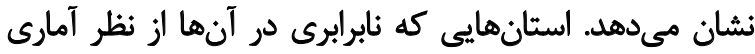

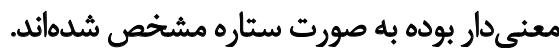

بحث

هدف مطالعه حاضر، بررسى وضعيت نابرابرى در استفاده از

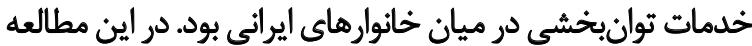

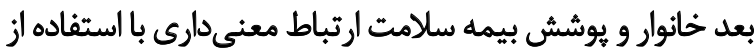

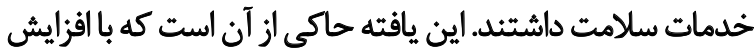

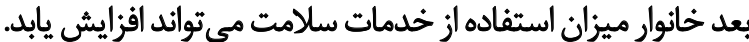

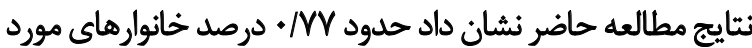

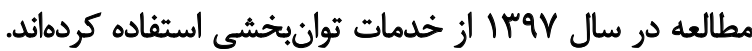

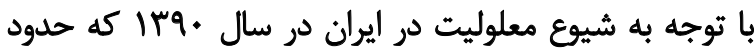

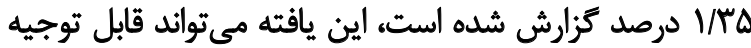

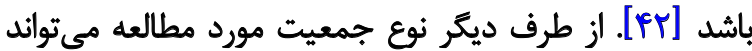

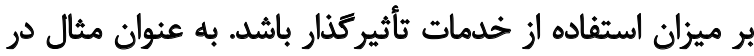
مطالعه ميلوشويج و همكاران كه روى بيماران مبتلا به مالتييل
كمترين نسبت استفاده را داشتند. بيشترين نسبت استفاده از

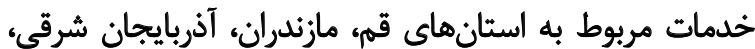

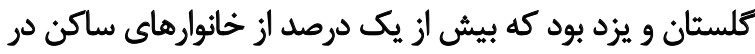

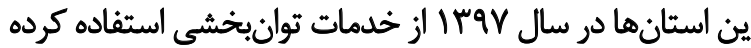

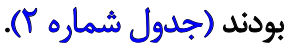
با توجه به توصيف تصويرى وضعيت استفاده از خدمات

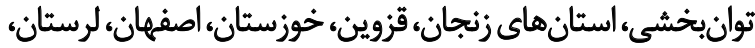

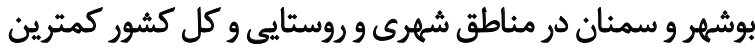

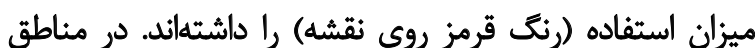

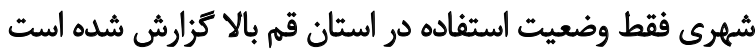

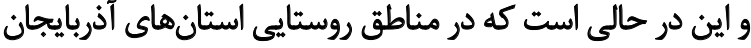

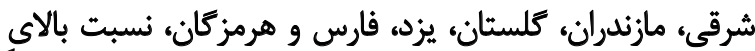

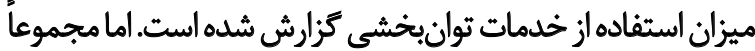

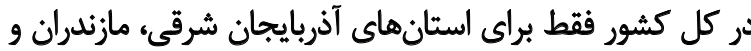

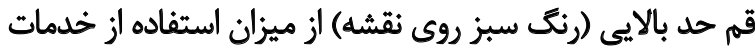
توانبخشى كزارش شده است (تصوير شماره ()).

مررسى نابرابرى در استفاده از خدمات توانبخشى در ايران نشان

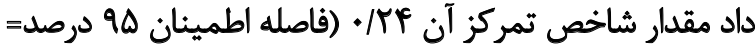

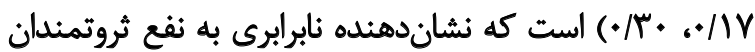

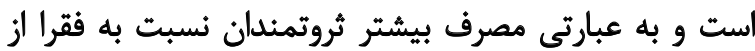

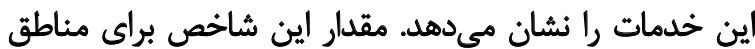

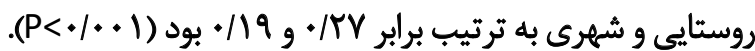

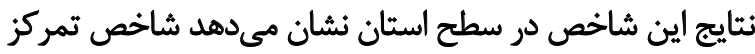

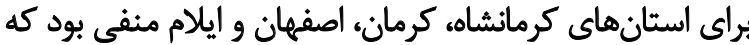

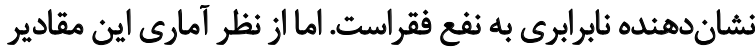

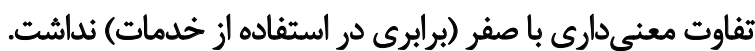




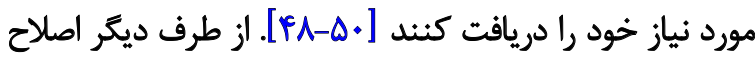

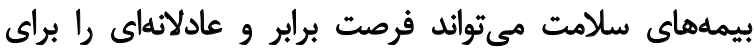

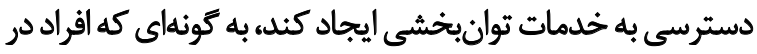

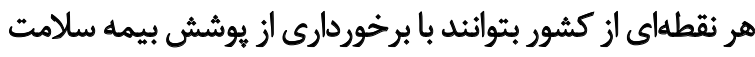
دسترسى مناسبترى به اين خدمات داشته باشند. براند

در مطالعه حاضر، استانهاى بوشهر و قم به طور معنى داردارى

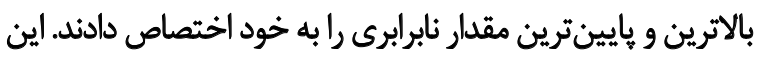

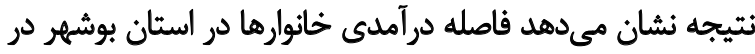

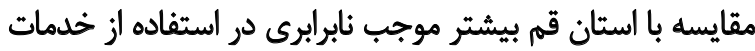

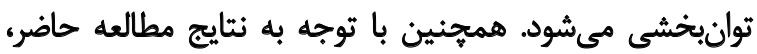

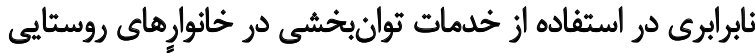

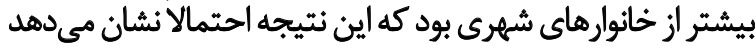

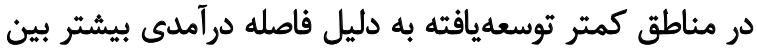

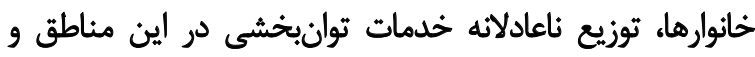

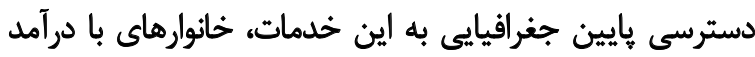

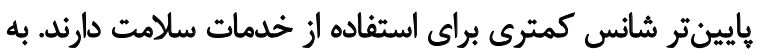

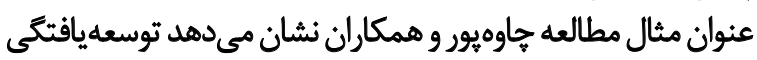

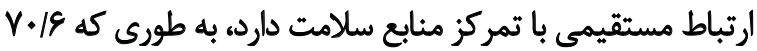

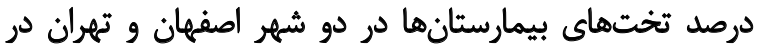

مناطق باوضعيت اجتماعى و اقتصادى بالاتر قرار داشتند [ـاندان.

علاوه بر اين، نتايج اين مطالعه نشان داد در ميان استانهاي مورد

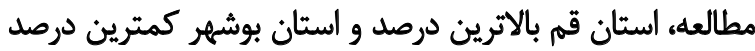

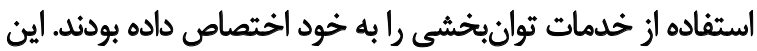

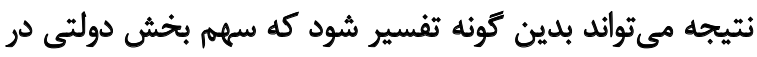

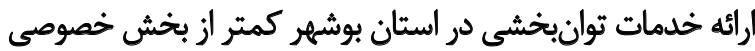

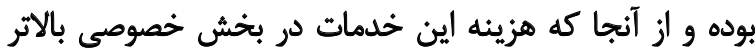

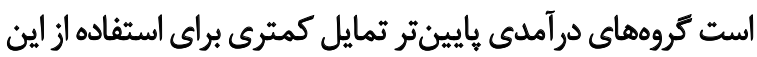

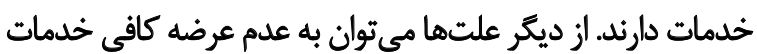

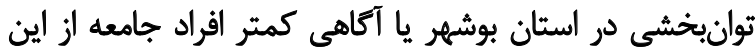

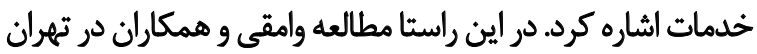

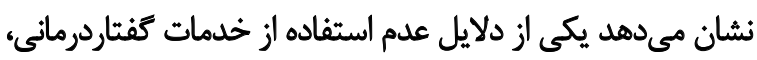

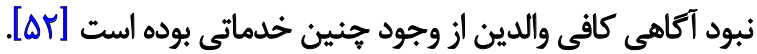

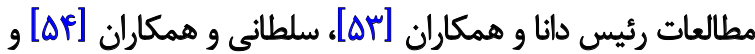

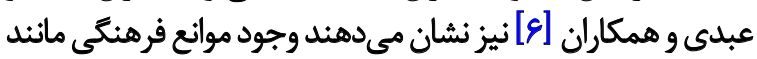

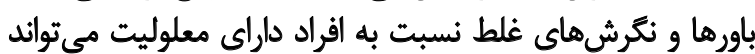
يكى از موانع استفاده از اين خدمات در ايران باشد.

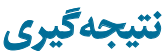

به طور كلى نتايج اين مطالعه نشان مى دهد نابرابرى درآمدى

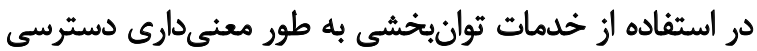

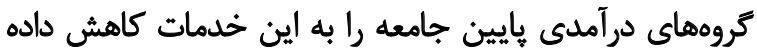

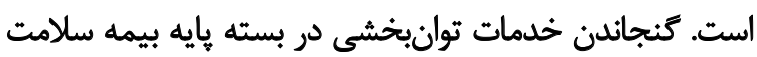

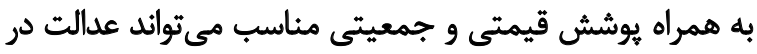

اسكلروزيس انجام شده است أf درصد بيماران از يك يا بي بيشتر

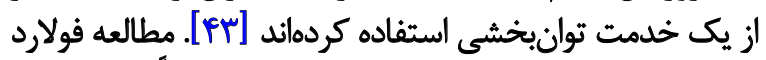

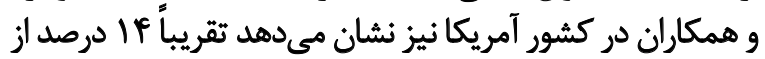

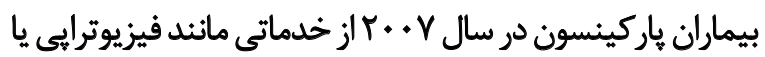

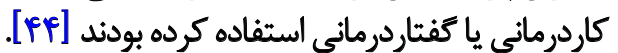

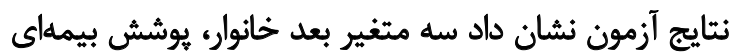

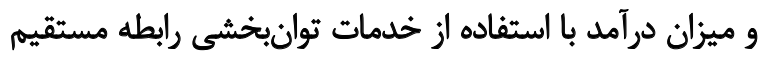

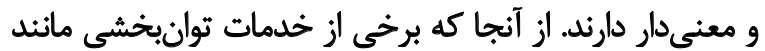

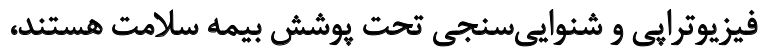

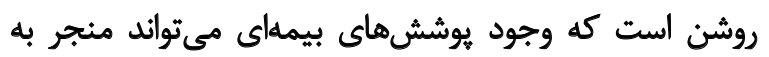

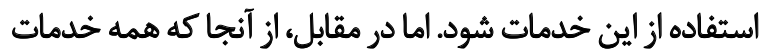

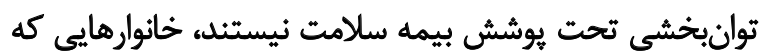

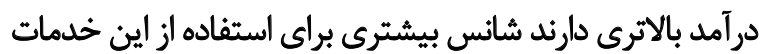

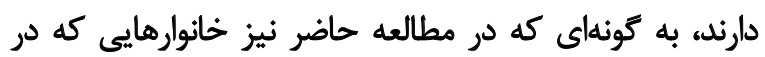

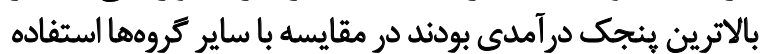
بيشترى از اين خدمات كرده بودند.

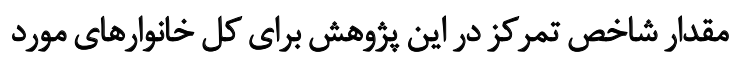

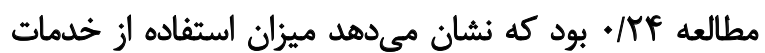

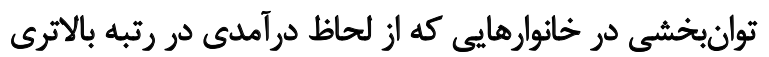

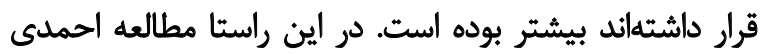

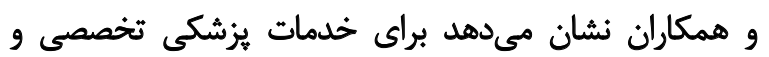

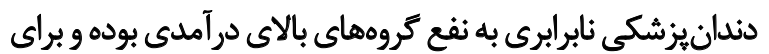

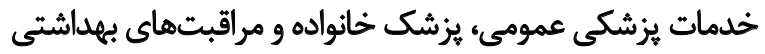

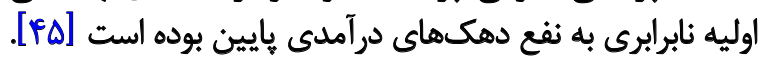

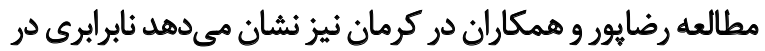

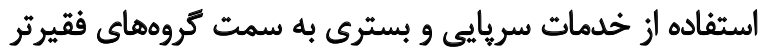

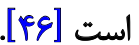

نبود يوششهاى بيمهاى براى برخى از خدمات توانبخشى

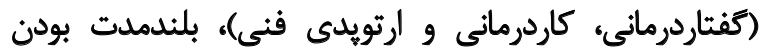

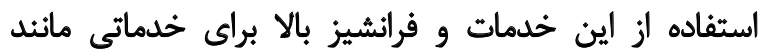

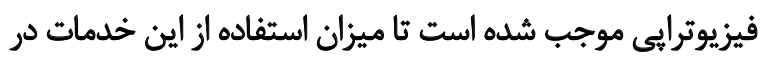

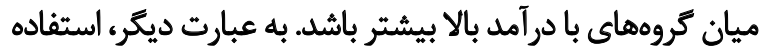

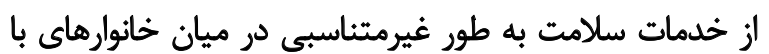

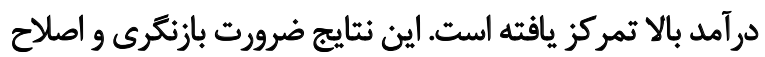

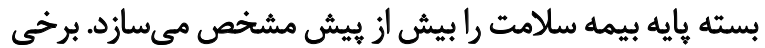

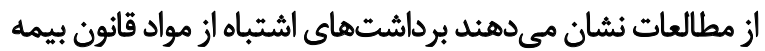

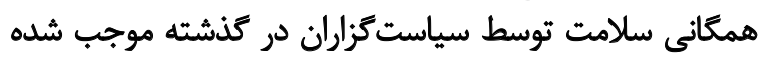

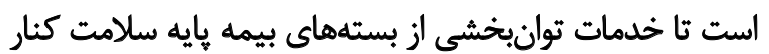

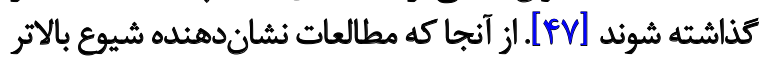

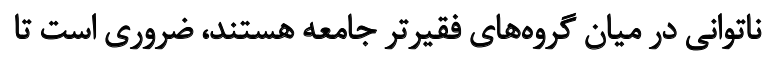

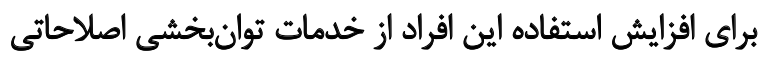

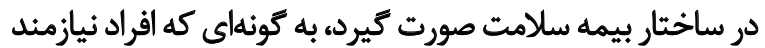

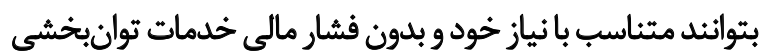




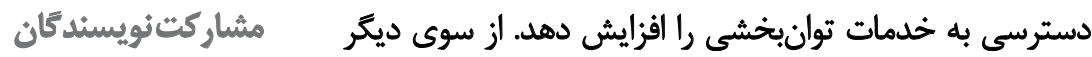
طراحى مطالعه: جعفر يحيوى ديزج، فاروق نعمانى، على

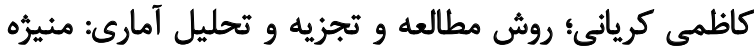

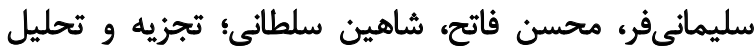

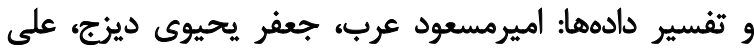

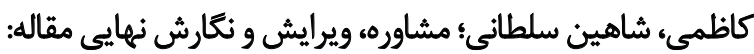

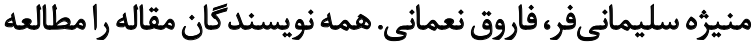
نمودهاند و محتواى آن را تأييد مىنمايند.

\section{تعارض مثافع}

بنا بر اظهارنامه نويسندكان، اين مقاله تعارض منافع ندارد.

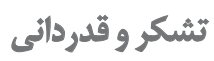

بدينوسيله از مركز آمار ايران جهت در دسترس قرار دادن

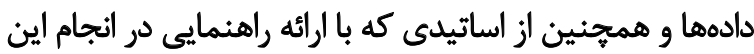

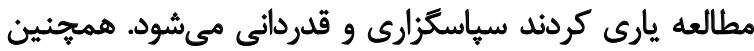

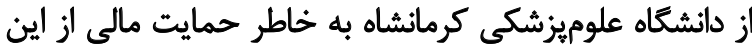

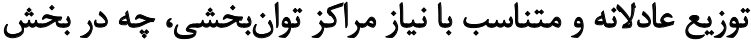

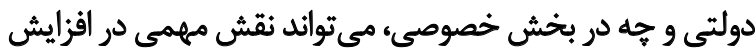

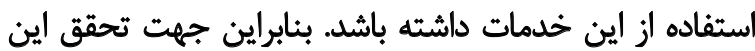

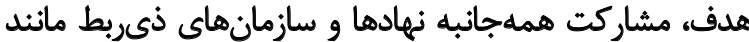

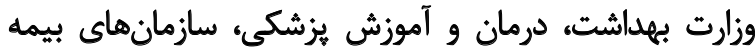

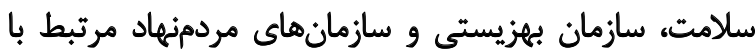

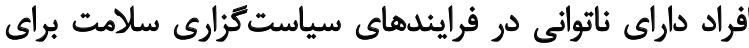

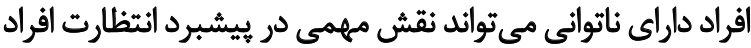

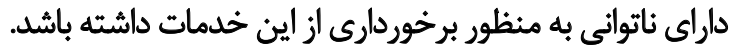

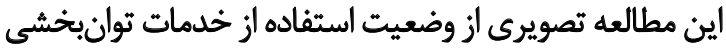

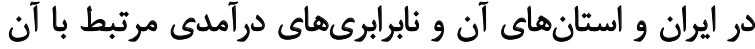

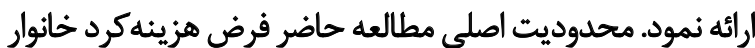

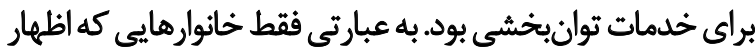

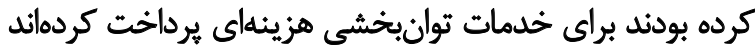

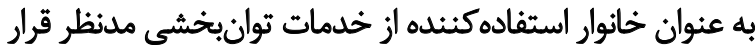

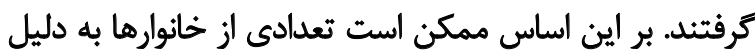

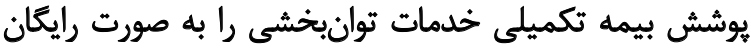

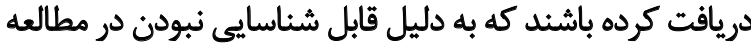

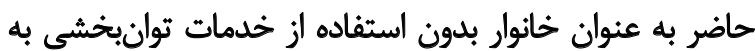

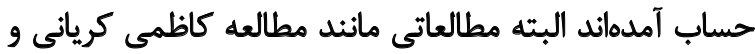

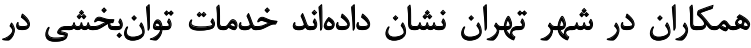

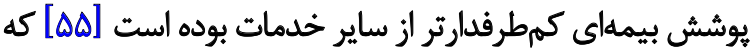

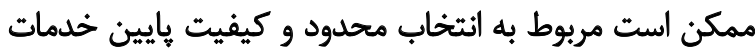

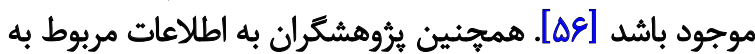

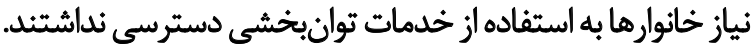

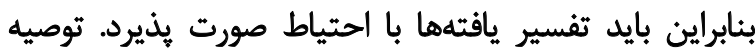

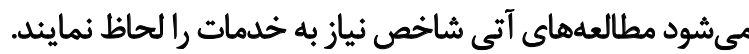
بيشنهاد مىشود اين مطالعه بر اساس ساير بايكاه دادهها نيز

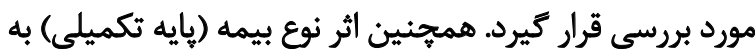

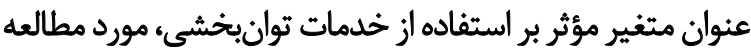

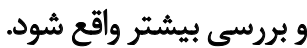

مالاحظاث اخلاقي

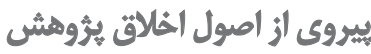

اين مطالعه توسط كميته اخلاق دانشَّاه علوم بزرشكى كرمانشاه تأييد شده است (كد: IR.KUMS.REC.1398.516).

$$
\text { حامى مالى }
$$

اين يُروهش تحت حمايت مالى دانشكاه علوميزشكى كرمانشاه

$$
\text { بوده است. }
$$




\section{References}

[1] Nooraiee Motlagh S, Saber Mahani A, Barooni M, Asadi Lari M, Vaez Mahdavi MR, Hadian M. [Determining factors related to health services utilization: Case of Tehran (Persian)]. Razi Journal of Medical Sciences. 2015; 21(127):61-72. http://rjms.iums.ac.ir/ article-1-3475-en.html

[2] Patwardhan B, Mutalik G, Tillu G. Integrative approaches for health: Biomedical research, ayurveda and yoga. San Diego, CA: Academic Press; 2015. [DOI:10.1016/C2013-0-19395-6]

[3] World Health Organization. Towards a common language for functioning, disability, and health ICF: The international classification of functioning, disability and health [Internet]. 2002 [Updated 2002]. Available from: https://www.who.int/classifications/icf/icfbeginnersguide.pdf

[4] Bright T, Wallace S, Kuper H. A systematic review of access to rehabilitation for people with disabilities in low-and middle-income countries. International Journal of Environmental Research and Public Health. 2018; 15(10):2165. [DOI:10.3390/ijerph15102165] [PMID] [PMCID]

[5] Bickenbach J. The world report on disability. Disability \& Society. 2011; 26(5):655-8. [DOI:10.1080/09687599.2011.589198]

[6] Abdi K, Arab M, Rashidian A, Kamali M, Khankeh HR, Khalajabadi Farahani F. Exploring barriers of the health system to rehabilitation services for people with disabilities in Iran: A qualitative study. Electronic Physician. 2015; 7(7):1476-85. [DOI:10.19082/1476] [PMID] [PMCID]

[7] Abdi K, Arab M, Khankeh HR, Kamali M, Rashidian A, Khalajabadi Farahani $\mathrm{F}$, et al. Challenges in providing rehabilitation services for people with disabilities in Iran: A qualitative study. Journal of Advances in Medicine and Medical Research. 2016; 13(4):1-11. https://www.journaljammr.com/index.php/JAM$\mathrm{MR} /$ article/view/13656

[8] Stucki G, Bickenbach J, Gutenbrunner C, Melvin J. Rehabilitation: The health strategy of the 21st century. Journal of Rehabilitation Medicine. 2018; 50(4):309-16. [DOI:10.2340/16501977-2200] [PMID]

[9] Adib Hajbagheri M. [Geriatic disability related factors (Persian)]. Salmand: Iranian Journal of Ageing. 2008; 3(2):547-55. http:// salmandj.uswr.ac.ir/article-1-89-en.html

[10] World Health Organization. The need to scale up rehabilitation [Internet]. 2017 [Updated 2017]. Available from: https://apps. who.int/iris/handle/10665/331210

[11] Nielsen NM, Kay L, Wanscher B, Ibsen R, Kjellberg J, Jennum P. Long-term socio-economic consequences and health care costs of poliomyelitis: A historical cohort study involving 3606 polio patients. Journal of Neurology. 2016; 263(6):1120-8. [DOI:10.1007/s00415-016-8108-1] [PMID]

[12] Cieza A, Chatterji S, McDaid D, Schmider A. Rehabilitation: Key for health in the 21st century. Paper presented at: Rehabilitation 2030: A Call for Action 2017. 6-7 February 2017; Executive Boardroom, WHO Headquarters, Geneva, Switzerland. https:// www.who.int/rehabilitation/rehab-2030-call-for-action/en/
[13] Grilli L, Feldman DE, Swaine B, Gosselin J, Champagne F, Pineault R. Wait times for paediatric rehabilitation. Healthcare Policy. 2007; 2(3):e171. [DOI:10.12927/hcpol.2007.18681]

[14] World Health Organization, World Bank. Summary: World report on disability 2011 [Internet]. 2011 [Updated 2011]. Available from: https://apps.who.int/iris/handle/10665/70670

[15] Karami Matin B, Kazemi Karyani A, Soltani Sh, Rezaei S, Soofi M. [Predictors of healthcare expenditure: Aging, disability or development? (Persian)] Archives of Rehabilitation. 2019; 20(4):310-21. [DOI:10.32598/ri.20.4.310]

[16] Turner-Stokes L, Williams H, Bill A, Bassett P, Sephton K. Cost-efficiency of specialist inpatient rehabilitation for workingaged adults with complex neurological disabilities: A multicentre cohort analysis of a national clinical data set. BMJ Open. 2016; 6(2):e010238. [DOI:10.1136/bmjopen-2015-010238] [PMID] [PMCID]

[17] Na'emani F, Esmaiil Zali M, Sohrabi Z, Fayaz-Bakhsh A. [Prevalence of risk factors for falls among the elderly receiving care at home (Persian)]. Salmand: Iranian Journal of Ageing. 2019; 13(5):638-51. [DOI:10.32598/SIJA.13.Special-Issue.638]

[18] Beswick AD, Rees K, Dieppe P, Ayis S, Gooberman-Hill R, Horwood J, et al. Complex interventions to improve physical function and maintain independent living in elderly people: A systematic review and meta-analysis. The Lancet. 2008; 371(9614):725-35. [DOI:10.1016/S0140-6736(08)60342-6]

[19] Iemmi V, Gibson L, Blanchet K, Suresh Kumar K, Rath S, Hartley $\mathrm{S}$, et al. Community-based rehabilitation for people with disabilities in low- and middle-income countries: A systematic review. Campbell Systematic Reviews. 2015; 11(1):1-177. [DOI:10.4073/ csr.2015.15]

[20] Soltani Sh, Takian AH, Akbari Sari A, Kamali M, Majdzadeh R, Karami Matin B. [Disregarded health problems of people with disabilities: A qualitative study of policymakers' perspective (Persian)]. Archives of Rehabilitation. 2019; 20(2):136-49. [DOI:10.32598/ri.20.2.136]

[21] UNICEF. Living conditions among persons with disability survey: Key findings report [Internet]. 2013 [Updated 2013]. Available from: https://atinfomap.org/downloads/National_Survey_ on_Disability_2013.pdf

[22] Kamaleri Y, Eide AH. Living conditions among people with disabilities in Lesotho: A national representative study [Internet]. 2011 [Updated 2011 February 20]. Available from: https://www. sintef.no/globalassets/sintef-teknologi-og-samfunn/rapportersintef-ts/report_lesotho_310111.pdf

[23] Eide AH, Kamaleri Y. Living conditions among people with disabilities in Mozambique: A national representative study [Internet]. 2009 [Updated 2009 January 12]. Available from: https:// www.sintef.no/globalassets/upload/helse/levekar-og-tjenester/ lc-report-mozambique-2nd-revision.pdf

[24] Loeb ME, Eide AH. Living conditions among people with activity limitations in Malawi: A national representative study [Internet]. 2004 [Updated 2004 August 26]. Available from: https://sintef. brage.unit.no/sintef-xmlui/bitstream/handle/11250/2461628/ STFA2477Living +Conditions+among+People+with+Activity+ Limitations + in + Malawi.pdf?isAllowed $=y \&$ sequence $=2$ 
[25] Srivastava DK, Khan JA, Pandey S, Pillai DS, Bhavsar AB. Awareness and utilization of rehabilitation services among physically disabled people of rural population of a district of Uttar Pradesh, India. International Journal of Medical Science and Public Health. 2014; 3(9):1157-60. [DOI:10.5455/ijmsph.2014.090720142]

[26] Gupta N, Castillo-Laborde C, Landry MD. Health-related rehabilitation services: Assessing the global supply of and need for human resources. BMC Health Services Research. 2011; 11:276. [DOI:10.1186/1472-6963-11-276] [PMID] [PMCID]

[27] Fathi F, Khezri A, Khanjani MS, Hosseinzadeh S, Abdi K. [Comparison of responsiveness status of rehabilitation services organization from the perspective of services recipients (Persian)]. Archives of Rehabilitation. 2019; 20(3):270-85. [DOI:10.32598/rj.20.3.270]

[28] Patel SK, Ladusingh L, editors. Age pattern of onset of disability and treatment seeking behaviour of disabled persons in India. Paper presented at: XXVI IUSSP International Population Conference. 27 September - 2 October 2009; Marrakesh, Morocco. https:// iussp2009.princeton.edu/abstracts/91173

[29] Borker S, Motghare D, Kulkarni M, Bhat Sh. Study of knowledge, accessibility and utilization of the existing rehabilitation services by disabled in a rural Goan community. Annals of Tropical Medicine and Public Health. 2012; 5(6):581-6. [DOI:10.4103/17556783.109284]

[30] Nketia-Kyere M, Aryeetey GC, Nonvignon J, Aikins M. Exploring barriers to accessing physiotherapy services for stroke patients at Tema general hospital, Ghana. Archives of Physiotherapy. 2017; 7:8. [DOI:10.1186/s40945-017-0037-5] [PMID] [PMCID]

[31] Ramirez M, Chang DC, Rogers SO, Yu PT, Easterlin M, Coimbra $\mathrm{R}$, et al. Can universal coverage eliminate health disparities? Reversal of disparate injury outcomes in elderly insured minorities. The Journal of Surgical Research. 2013; 182(2):264-9. [DOI:10.1016/j. jss.2012.01.032] [PMID]

[32] Englum BR, Villegas C, Bolorunduro O, Haut ER, Cornwell III EE, Efron DT, et al. Racial, ethnic, and insurance status disparities in use of posthospitalization care after trauma. Journal of the American College of Surgeons. 2011; 213(6):699-708. [DOI:10.1016/j.jamcollsurg.2011.08.017] [PMID] [PMCID]

[33] de la Plata CM, Hewlitt M, de Oliveira A, Hudak A, Harper C, Shafi Sh, et al. Ethnic differences in rehabilitation placement and outcome after TBI. Journal of Head Trauma Rehabilitation. 2007; 22(2):11321. [DOI:10.1097/01.HTR.0000265099.29436.56] [PMID]

[34] Meagher AD, Beadles CA, Doorey J, Charles AG. Racial and ethnic disparities in discharge to rehabilitation following traumatic brain injury. Journal of Neurosurgery. 2015; 122(3):595-601. [DOI:10.3171/2014.10.JNS14187] [PMID]

[35] Sacks GD, Hill C, Rogers Jr SO. Insurance status and hospital discharge disposition after trauma: inequities in access to postacute care. The Journal of Trauma: Injury, Infection, and Critical Care. 2011; 71(4):1011-5. [DOI:10.1097/TA.0b013e3182092c27] [PMID]

[36] McNamara A, Normand C, Whelan B. Patterns and determinants of health care utilisation in Ireland [Internet]. 2013 [Updated 2013 February]. Available from: https://tilda.tcd.ie/publications/reports/ HealthcareUtilisation/ [DOI:10.38018/TildaRe.2013-00]

[37] Haider AH, Efron DT, Haut ER, DiRusso SM, Sullivan T, Cornwell III EE. Black children experience worse clinical and functional outcomes after traumatic brain injury: An analysis of the National Pediatric Trauma Registry. The Journal of Trauma: Injury, Infection, and Critical Care. 2007; 62(5):1259-63. [DOI:10.1097/TA.0b013e31803c760e] [PMID]

[38] Wood JN, Hall M, Schilling S, Keren R, Mitra N, Rubin DM Disparities in the evaluation and diagnosis of abuse among infants with traumatic brain injury. Pediatrics. 2010; 126(3):408-14. [DOI:10.1542/peds.2010-0031] [PMID]

[39] Akande M, Minneci PC, Deans KJ, Xiang H, Chisolm DJ, Cooper JN. Effects of Medicaid expansion on disparities in trauma care and outcomes in young adults. The Journal of Surgical Research. 2018; 228:42-53. [DOI:10.1016/j.jss.2018.02.058] [PMID]

[40] Hicks CW, Hashmi ZG, Velopulos C, Efron DT, Schneider EB, Haut ER, et al. Association between race and age in survival after trauma. JAMA Surgery. 2014; 149(7):642-7. [DOI:10.1001/jamasurg.2014.166] [PMID] [PMCID]

[41] Wagstaff A. The bounds of the concentration index when the variable of interest is binary, with an application to immunization inequality. Health Economics. 2005; 14(4):429-32. [DOI:10.1002/ hec.953] [PMID]

[42] Soltani Sh, Khosravi B, Salehiniya H. Prevalence of disability in Iran. Iranian Journal of Public Health. 2015; 44(10):1436-7. https://ijph.tums.ac.ir/index.php/ijph/article/view/5015

[43] Milivojević I, Adamec I, Habek M. Utilization of physical rehabilitation among people with multiple sclerosis. Irish Journal of Medical Science. 2013; 182(3):429-32. [DOI:10.1007/s11845 013-0905-2] [PMID]

[44] Fullard ME, Thibault DP, Hill A, Fox J, Bhatti DE, Burack MA, et al. Utilization of rehabilitation therapy services in Parkinson disease in the United States. Neurology. 2017; 89(11):1162-9. [DOI:10.1212/WNL.0000000000004355] [PMID] [PMCID]

[45] Ahmadi AM, Nikravan A, Naseri AR, Asari A. [Effective determinants in household out of packet payments in health system of Iran, using two part regression model (Persian)]. Journal of Health Administration. 2014; 17(56):7-18. http://jha.iums.ac.ir/ article-1-1469-en.html

[46] Rezapoor A, Roumiani Y, Ebadifard Azar F, Ghazanfari S, Mirzaei S, Sarabi Asiabar A, et al. [Effective factors on utilization and access to health care: A population-based study in Kerman (Persian)]. Journal of Health Administration. 2015; 18(60):24-36. http://jha.iums.ac.ir/article-1-1636-en.html

[47] Soltani Sh, Takian AH, Akbari Sari A, Majdzadeh R, Kamali M. Financial barriers to access to health services for adult people with disability in Iran: The challenges for universal health coverage. Iranian Journal of Public Health. 2019; 48(3):508-15. [DOI:10.18502/ijph.v48i3.895] [PMID] [PMCID]

[48] Lustig DC, Strauser DR. Causal relationships between poverty and disability. Rehabilitation Counseling Bulletin. 2007; 50(4):194 202. [DOI:10.1177/00343552070500040101]

[49] Palmer M. Disability and poverty: A conceptual review Journal of Disability Policy Studies. 2011; 21(4):210-8, [DOI:10.1177/1044207310389333] 
[50] Yeo R. Chronic poverty and disability [Internet]. 2001 [Updated 2001 August 1]. Available from: https://ssrn.com/abstract $=1754542$ [DOI:10.2139/ssrn.1754542]

[51] Chavehpour Y, Rashidian A, Woldemichael A, Takian AH. Inequality in geographical distribution of hospitals and hospital beds in densely populated metropolitan cities of Iran. BMC Health Services Research. 2019; 19(1):614. [DOI:10.1186/s12913-0194443-0] [PMID] [PMCID]

[52] Ali-Abadi S, Hatami-Zadeh N, Vameghi R, Kazem-Nezhad A. Disabled children and home exercises: Barriers to compliance with recommendations of therapists (Persian)]. Archives of Rehabilitation. 2012; 13(1):39-46. http://rehabilitationj.uswr.ac.ir/ article-1-830-en.html

[53] Raeis-Dana M, Tabatabaei-Nia M, Kamali M, Shafaroudi N. [From diagnosis to coping: A journey with parents in the course of the disability of their children (Persian)]. Journal of Rehabilitation. 2009; 10(1):42-51. http://rehabilitationj.uswr.ac.ir/article1-313-en.html

[54] Soltani Sh, Takian AH, Akbari Sari A, Majdzadeh R, Kamali M. Cultural barriers in access to healthcare services for people with disability in Iran: A qualitative study. Medical Journal of the Islamic Republic of Iran. 2017; 31(1):293-9. [DOI:10.14196/ miri.31.51] [PMID] [PMCID]

[55] Kazemi Karyani A, Akbari Sari A, Woldemichael A. Eliciting preferences for health insurance in Iran using discrete choice experiment analysis. International Journal of Health Policy and Management. 2019; 8(8):488-97. [DOI:10.15171/ijhpm.2019.29] [PMID] [PMCID]

[56] Ahmadzadeh N, Ebadi Fard Azar F, Baradaran HR, Salman Roghani R, Shirazikhah M, Joghataei MT, et al. Availability of outpatient rehabilitation facilities in 2015 in Iran: A nationwide study. Medical Journal of The Islamic Republic of Iran. 2018; 32(1):721-6. [DOI:10.14196/mjiri.32.123] [PMID] [PMCID] 